\title{
Sequential Reporting Bias
}

August 14, 2021

\begin{abstract}
Firms with correlated fundamentals often issue reports sequentially, leading to information spillovers. The theoretical literature has investigated multi-firm reporting, but only when firms report simultaneously. We examine the implications of sequential reporting, where firms aim to maximize their market price and can manipulate their reports. Our model demonstrates that the introduction of sequentiality in the presence of information spillovers significantly alters the biasing behavior of firms and the resulting informational environment relative to simultaneous reporting. In particular, a lead firm always manipulates more when reports are issued sequentially. Interestingly, this occurs because follower firms, who benefit from information spillovers, place less weight on their own private information when issuing a report. This information loss leads the market to place greater weight on the leaders report, which increases the incentive of the lead manager to manipulate her report. Moreover, the information loss from sequentiality leads to less efficient and less volatile prices. Additionally, we find that stronger correlation in firm fundamentals can amplify the lead firm's incentive for manipulation under sequentiality, in contrast to simultaneous reporting. We offer additional results regarding, for example, market response coefficients, and provide a number of empirical implications.
\end{abstract}

Keywords: Disclosure, information spillovers, reporting, manipulation, bias, clustering. JEL Classification: C72, D82, D83, G14, M41. 


\section{Introduction}

Informational spillovers are a pervasive feature of financial markets. Firms public dissemination of information allows competitors and other market participants to learn relevant information regarding industry or market conditions. For example, a firms earnings announcement or management forecast can convey important industry-level information regarding future product demand, risk exposure, or access to credit or equity. Indeed, the notion that firms can benefit from observing the information released by their peers has been well documented in the empirical literature. ${ }^{1}$ The presence of sequential learning by firms may also affect managerial incentives to distort their reports. However, the extant theoretical literature considering manipulation in reporting has only examined single-firm settings, or multi-firm settings where reporting is simultaneous, and thus does not capture the interplay between manipulation and sequential peer-learning by firms. ${ }^{2}$ The goal of this paper is to explore managerial incentives and the equilibrium properties of reporting when firms release information sequentially. In doing so, we show the distinct incentives and equilibrium characteristics that arise when firms can learn from each other through sequential reporting, and we provide a direct comparison of these incentives under sequentiality to a simultaneous reporting regime.

Our model is designed to capture the following important features of the corporate reporting environment. First, we capture information spillovers by assuming that firm fundamentals are correlated and managers can learn about their own firms value by observing the report of their peer. Second, managers can manipulate their reports, but they incur a cost from deviation of the report from their firms true value. As such, each managers expected cost is lower when she obtains more precise information and when she manipulates the reports less. Managers seek to maximize their firms market price net of their costs from deviation of the report from the firms true value. A firms price is set by risk-neutral investors to equal the expected firm value based on both firms' reports. In addition to unobservability

\footnotetext{
${ }^{1}$ These include, for example, Foster (1981), Baginski (1987), Han et al. (1989), Freeman and Tse (1992), Ramnath (2002), Thomas and Zhang (2008), Tse and Tucker (2010), Pandit et al. (2011), Brochet et al. (2018), Gong et al. (2019), Hann et al. (2019), and Truong (2019).

${ }^{2}$ In the context of earnings announcements, a number of studies find evidence consistent with laterreporting firms adjusting earnings numbers following the announcements of industry leaders, such as Kedia et al. (2015), Bratten et al. (2016), Gong et al. (2019), and Kim et al. (2019). Indeed, Gong et al. (2019) note that: "In our sample, the average reporting lag in earnings releases between accounting-based RPE firms and their early-announcing peers is about two weeks. This time window is sufficient for managers to deliberate last-minute accounting adjustments necessary to achieve (estimated) target performance. As noted in PricewaterhouseCoopers (2010), 'companies are able to produce consolidated reports within five business days ... [and in] many cases, this accelerated cycle is followed by a series of post-close adjusting entries that continue up to the release of earnings.' These anecdotal observations suggest that accounting adjustments are common and can be quickly approved by auditors prior to earnings releases" (p. 361).
} 
of the managers' private noisy signals, investors face some uncertainty regarding the managers' objectives (e.g., Heinle and Verrecchia (2016)). ${ }^{3}$ The additional layer of information asymmetry results in the realistic feature that a managers report does not fully reveal her private information.

We first fully analyze the unique linear equilibrium of the sequential reporting setting, which includes each manager's reporting strategy and the market's pricing function. We also derive the equilibrium of the simultaneous reporting setting and compare it to the sequential regime to better understand and highlight how sequentiality in reporting affects various features of the equilibrium. Our parsimonious setting provides a number of novel results. These include results regarding: $(i)$ managerial reporting bias - how sequentiality in reporting affects manipulation incentives; $(i i)$ informational environment - the informational implications of sequential as compared to simultaneous reporting, and the market response coefficients in each reporting regime; and (iii) managers' timing preferences - conditions under which managers prefer reporting simultaneously relative to sequentially.

Our first main result shows that an industry reporting leader always biases her report more under a sequential reporting regime relative to a simultaneous regime (Theorem 1). In other words, a firm that reports first in the sequential reporting setting always biases more than in the simultaneous regime where informational spillovers are absent. This implies that the sequential nature of reporting has a direct effect on the biasing behavior of firms. Moreover, this result implies cross-industry variation in the level of misreporting by firms. In particular, in industries where firms' information releases are dispersed, we expect to see greater levels of manipulation in the reports of early movers, as compared to industries with clustered releases.

To understand the economic forces driving this result, first note that, in the simultaneous reporting regime, information spillovers play no direct role in the reporting behavior of managers, and each manager relies only on her own private information when forming beliefs and issuing a report. In contrast, in the sequential regime, information spillovers from the first (lead) managers report become salient. The second manager (the follower) incorporates the relevant information from the first report when forming beliefs of her own firm's value. As a result, the follower relies relatively less on her own private information when forming beliefs and issuing her report. This lower reliance by the follower on her private information decreases the informativeness of the second report, and leads to less overall information that is obtained by the market under sequentiality than in the simultaneous regime. Due

\footnotetext{
${ }^{3}$ Specifically, we assume that investors do not perfectly know all of the parameters of the managers biasing costs. Our results are qualitatively unchanged if, instead of assuming uncertainty about the managers' biasing costs, we were to assume that the market observes each manager's report with some noise (as in Versano and Trueman (2017)).
} 
to this information loss in the followers report, the market places greater weight on the lead manager's report when forming beliefs regarding the firms' values. That is, the price of the lead firm becomes more sensitive to its report, which amplifies the lead manager's incentive to manipulate her report. As a result, the bias of the lead manager's report is greater compared to her bias in the simultaneous reporting regime.

We note that this result is quite general in the sense that it always holds in our setting, even though we allow firms to be heterogeneous in all parameters. Moreover, as indicated above, the fact that the follower manager relies less on her private information leads to overall information loss to the market. This implies that, under sequentiality, the market is facing higher uncertainty, and thus prices are less informative about the firms values. Another implication is that, since reports convey less information in the sequential regime, prices should exhibit lower volatility compared to the simultaneous regime. In order to highlight the potential real effects of information loss under sequential reporting, we offer an extension of the model that considers a project decision that is made by the managers after the reports are issued (Section 6.3).

We additionally examine the bias of the follower manager under both reporting regimes. We identify a necessary and sufficient condition under which the follower manager's report exhibits a greater bias as compared to the simultaneous regime. As we explain in more detail in the analysis, there are two opposing effects that determine the magnitude of the second manager's bias. On one hand, since the report of the follower manager contains less information compared to the simultaneous regime, it makes the price less sensitive to her report. This information loss effect decreases the managers incentive to bias her report. On the other hand, the fact that the follower manager assigns a lower weight to her private information about firm value in forming the report scales down the variation of the report. To undo this decrease in the variation of the report, the market pricing becomes more responsive to the managers report (keeping all else equal). This scaling effect increases the manager's incentive to bias. Depending on which effect dominates, the follower manager's bias will be either higher or lower than in the simultaneous regime.

We explore equilibrium properties regarding the managers' manipulation incentives, reporting strategies, and response coefficients. In particular, we consider the change in manipulation levels as we increase the correlation between firm values. Under the simultaneous regime, greater correlation between firms implies that each firm's individual report becomes relatively less important for its own pricing, as the firm's peer report becomes more informative. This reduces the incentive to manipulate for each firm. This effect has been documented in previous studies which feature simultaneous reporting, such as Strobl (2013) and Heinle and Verrecchia (2016). However, when firms report sequentially, the follower 
relies more heavily on the leader's report as the correlation in firm value increases. This exacerbates the information loss in the follower's report under sequentiality, which amplifies the market's weight on the lead manager's report. Consequently, when the follower's benefit from informational spillovers is sufficiently high, the lead manager's incentive to manipulate increases, resulting in greater manipulation as the correlation increases. This property is a novel insight of sequentiality and contrasts with the extant literature that studies simultaneous reporting. Moreover, this property highlights implications which emerge from our comparative analysis regarding variation among industries that exhibit staggered reporting.

We additionally consider the managers preferences regarding the timing of their reports. In particular, we examine when both managers would prefer simultaneous relative to sequential reporting. We find that both managers prefer simultaneous reporting when the cost to misreporting and uncertainty regarding the managers' objectives are sufficiently low, so that the market is sufficiently adept at interpreting the manager's report (Theorem 2). As noted above, a manager that reports second and observes the report of her peer always benefits from informational rents relative to reporting simultaneously. However, the overall manipulation cost of being a follower may also be higher. Indeed, when the market is better able to extract the manager's private information from the report, the second manager's cost from manipulation overwhelms the benefits from information spillovers. As a result, neither manager prefers to delay their report and become a follower. These results may have implications regarding which industries should exhibit clustering in the timing of information release by firms.

Several empirical implications emerge from our analysis. As discussed above, we expect to see greater bias among industry reporting leaders under sequential rather than simultaneous reporting. In addition, within industries with sequential reporting, we expect to see greater manipulation by follower firms in industries where the market's inference of reports is more precise, such as in less complex industries. Relatedly, due to the information loss that emerges from sequential reporting, later reports are less informative than early reports, consistent with the empirical findings of Givoly and Palmon (1982). Additionally, the results predict that early reports play an outsized role and disproportionately influence market beliefs in industries with sequential reporting. Furthermore, the presence of sequentiality has implications for price efficiency. As the market suffers a net information loss under sequential reporting, we expect to observe less efficient prices - where the efficiency loss applies to all firms - and greater information asymmetry between firms and the market.

Importantly, a central feature of our setting is that firms can be heterogeneous in all parameters. One benefit of this structure is that it allows us to develop sharp predictions concerning how the characteristics of a firm's peers affect the firm's biasing behavior. In 
other words, our setting gives rise to predictions concerning peer effects in firm misreporting. The study of peer effects among firms in capital markets is of recent interest in the empirical literature (e.g., Leary and Roberts (2014), Kaustia and Rantala (2015), Grennan (2019), Seo (2020)). Our results predict that firms exhibit greater manipulation in their reports when their industry peers have, on average, less severe information asymmetry between the firm and investors before reports are issued, $(i i)$ less accurate private information; or ( $i i i$ ) lower market inference of reports, such as more complex releases. We note that this prediction is quite general in the sense that it holds under both reporting regimes (sequential and simultaneous), and holds regardless of the firm's reporting position (in the case of sequential reporting).

Our results also have implications for the reporting patterns across industries. We find that firms prefer simultaneous reporting when the markets inference of the manager's information from reports is more precise and when manipulation costs are low. Such industries are more likely to follow a simultaneous reporting regime. Likewise, industries where managers have greater difficulty in misreporting, such as industries with better corporate governance, more regulatory burdens, greater oversight, or where managers have less discretion and control, may be more likely to exhibit sequential reporting. While a number of studies have found evidence consistent with strategic timing of forecasts and announcements (e.g., Givoly and Palmon (1982), Skinner (1994), Kothari et al. (2009), Tse and Tucker (2010), deHaan et al. (2015), Gong et al. (2019), Truong (2019)), cross-industry variation in the reporting patterns of firms - sequential versus simultaneous - has not been fully explored in the extant empirical literature. Our results may therefore help to guide future empirical investigation. These predictions, as well as others, are more thoroughly discussed in Section 5.

\subsection{Related Literature}

Our model relates to the theoretical literature that investigates reporting manipulation among multiple firms with correlated fundamentals. Strobl (2013) considers manipulation when firm value is correlated with a systematic risk factor. Heinle and Verrecchia (2016) consider reporting biases among multiple firms, where the number of firms that commit to disclose is determined endogenously. Einhorn, Langberg, and Versano (2018) examine reporting by two firms in a Cournot setting where managers can alter real production decisions to influence the reports (performance) of rival firms. Gao and Zhang (2019) study a model where the ex ante manipulation decision is an endogenous strategic complement across firms, and find that firms under-invest in internal controls. A distinctive feature shared by all of these models is that firms are assumed to report simultaneously. Hence, our models focus 
on sequentiality provides new insights regarding how the potentially staggered reporting of firms has a direct effect on firm biasing behavior and the markets total information relative to simultaneous reporting.

Trueman (1990) analyzes strategic earnings announcement timing where a (follower) firm can delay information to lower the cost of manipulation. The present study varies from Trueman (1990) as we compare manipulation incentives between two distinct reporting regimes. Moreover, we examine the biasing incentives for all firms in our setting, including the reporting leader, whereas the leader is nonstrategic in Trueman (1990). Our model is also related to the literature which studies manipulation in reporting in single-firm settings, such as Trueman and Titman (1988), Fischer and Verrecchia (2000), Kirschenheiter and Melumad (2002), Ewert and Wagenhofer (2005), Guttman, Kadan, and Kandel (2006), Chen, Hemmer, and Zhang (2007), Caskey, Nagar, and Petacchi (2010), Friedman (2014), and Bertomeu, Darrough, and Xue (2017), among others. Our paper adds to this literature by considering how the sequential nature of reporting by multiple firms can influence manipulation incentives.

Our results also contribute to the literature examining information spillovers and observational learning in capital markets (e.g., Persons and Warther (1997), Altı (2005), Aghamolla and Guttman (2020)). Jorgensen and Kirschenheiter (2012) consider a sequential-move costly voluntary disclosure model (à la Jovanovic (1982) and Verrecchia (1983)) where the leader's disclosure can benefit the follower by allowing the follower to save disclosure costs. Our model varies in that we allow managers to manipulate their reports, and we compare incentives between sequential and simultaneous reporting. Moreover, our study broadly contributes to the social learning literature (e.g., Banerjee (1992), Bikhchandani et al. (1992)) in two important ways. First, we allow agents to manipulate their observable actions. Second, we assume that each agent's private information is imperfectly revealed to the market and other agents. This leads to sharp implications regarding price efficiency and the manipulation behavior of agents in a setting with information spillovers (sequential reporting) compared to one without such learning (simultaneous reporting).

The remainder of this paper is structured as follows. The following section presents the model. In Section 3, we examine the equilibrium under sequential reporting and compare it to a benchmark case of simultaneous reporting. Section 4 investigates properties of equilibrium, and Section 5 discusses empirical predictions. Extensions are discussed in Section 6 and the final section concludes. All proofs are relegated to the Appendix. 


\section{Model}

We consider a setting with two firms whose managers communicate, such as through a report or forecast, their firm's performance to a risk-neutral capital market. Each manager, $i=1,2$, privately observes an imperfect signal, denoted by $s_{i}$, of her respective firm's value, denoted by $\theta_{i}$. Manager $i$ 's private signal is given as:

$$
s_{i}=\theta_{i}+\varepsilon_{i},
$$

where $\theta_{i}$ is normally distributed with mean zero and precision $\tau_{i}^{\theta}$ (i.e., the variance is $\left.1 / \tau_{i}^{\theta}\right){ }^{4}$ The parameter $\varepsilon_{i}$ is a normally distributed error term with mean zero and precision $\tau_{i}^{\varepsilon}$ that is independent of $\theta_{i}, \theta_{-i}$, and $\varepsilon_{-i} .{ }^{5}$ The information structure is such that the values of the two firms $\theta_{1}$ and $\theta_{2}$ are correlated according to correlation parameter $\rho \in(-1,1) .{ }^{6}$ In particular, the variance-covariance matrix for the vector $\left(\theta_{1} \theta_{2}\right)^{\prime}$ is given as

$$
\Sigma_{\theta} \equiv\left(\begin{array}{cc}
\frac{1}{\tau_{1}^{\theta}} & \frac{\rho}{\sqrt{\tau_{1}^{\theta} \tau_{2}^{\theta}}} \\
\frac{\rho}{\sqrt{\tau_{1}^{\theta} \tau_{2}^{\theta}}} & \frac{1}{\tau_{2}^{\theta}}
\end{array}\right)
$$

Each manager provides a report $r_{i}$ to the market. Our primary interest is in the sequential regime. In this case, manager 1 (often referred to as the "leader") issues her report $r_{1}$ in stage one, and manager 2 (the "follower") issues $r_{2}$ in stage two after observing $r_{1}$. In stage three, the risk-neutral market prices both firms. We denote the price of firm $i$ as $P_{i} \equiv \mathbb{E}\left[\theta_{i} \mid r_{1}, r_{2}\right] .^{7}$ This sequential setup captures the notion of informational spillovers by firm reports in financial markets (e.g., Freeman and Tse (1992), Tse and Tucker (2010), Truong (2019)). Moreover, as noted previously, a number of studies find evidence consistent with follower firms adjusting their reports after observing the report of a lead firm, such as Kedia et al. (2015), Bratten et al. (2016), Gong et al. (2019), and Kim et al. (2019).

To provide additional texture to our results, we often compare this sequential regime to a benchmark setting where managers report simultaneously, i.e., the simultaneous regime. In

\footnotetext{
${ }^{4}$ The mean zero assumption on $\theta_{i}$ is without loss of generality.

${ }^{5}$ We use the subscript $-i$ to denote terms corresponding to the firm other than firm $i$.

${ }^{6}$ Alternatively, we may allow the link in fundamentals to occur through a common component between firms, whereby each firm's value is the sum of an idiosyncratic component, $v_{i}$, and a common component $\phi$, i.e., $\theta_{i}=v_{i} \pm \phi$. A disclosure by one firm also provides information about the common component $\phi$. When the sign in front of $\phi$ is the same (resp. not the same) for both firms, the common factor model is equivalent to our model with $\rho>0$ (resp. $\rho<0$ ).

${ }^{7}$ Allowing managers to also be concerned about market beliefs immediately after issuing their report (e.g., $\mathbb{E}\left[\theta_{i} \mid r_{1}\right]$ for manager 1 ) does not substantively affect the results. We analyze the presence of short-term price on incentives in Section 6.2.
} 
this benchmark case, managers 1 and 2 issue their reports simultaneously in the same stage, and the market prices the firms based on the two reports.

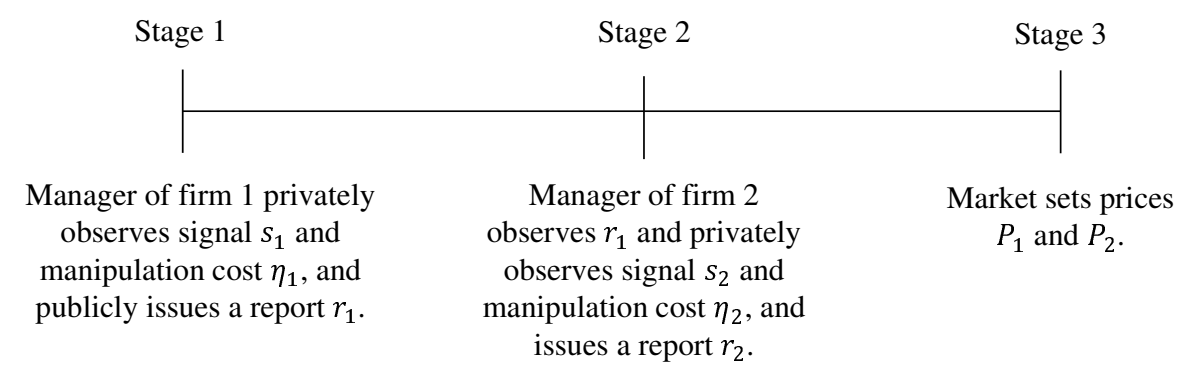

Figure 1: Timeline of the sequential regime.

We assume that managers care about the accuracy of their reports to the market. This can capture, for instance, the manager's reputational concerns regarding the market's assessment of her ability. For example, Goodman et al. (2013) find that managers who issue more accurate forecasts also make more profitable investment decisions. Likewise, Graham et al. (2005) note that managers with inaccurate reports may be perceived as poorly running the firm. ${ }^{8}$ We also assume that managers can distort their reports, however such manipulation is personally costly. We capture both of these features parsimoniously in the following disutility function:

$$
\frac{c_{i}\left(r_{i}-\theta_{i}-\eta_{i}\right)^{2}}{2}
$$

where $\eta_{i} \sim N\left(0,1 / \tau_{i}^{\eta}\right)$ is manager $i$ 's privately observed manipulation cost parameter (as in, e.g., Dye and Sridhar (2008), Beyer (2009) and Beyer, Guttman, and Marinovic (2019)). This can be interpreted, for example, as adjustments made in the report to comply with the firm's accounting rules or with the auditor's or other stakeholders' interests, ${ }^{9}$ or other idiosyncratic circumstances that affect the manager's ability to misreport. The additional information asymmetry introduced through $\eta_{i}$ leads the manager's private information $s_{i}$ to be imperfectly recovered from the report $r_{i}$. As such, the market can more accurately update its beliefs concerning $s_{i}$, and thus $\theta_{i}$, when the precision of $\eta_{i}\left(\tau_{i}^{\eta}\right)$ is higher. Hence, we often refer to $\tau_{i}^{\eta}$ as the precision of the market's inference of $s_{i}$ from the report $r_{i}$. We note that an alternative specification which is equivalent and yields the same results is where there is no uncertainty about the manager's objective function, but the market observes the

\footnotetext{
${ }^{8}$ In particular, Graham et al. (2005) note that "[...] if the firm had previously guided analysts to the EPS target, then missing the target can indicate that a firm is managed poorly in the sense that it cannot accurately predict its own future" (p. 5).

${ }^{9}$ Such adjustments may not be perfectly understood by investors due to the complexity of accounting rules. See, e.g., Chychyla et al. (2019).
} 
manager's report with noise (e.g., Versano and Trueman (2017)). For example, the market may have greater difficulty in making precise inferences (i.e., $\tau_{i}^{\eta}$ is lower) in industries which are more complex or have more complicated information releases (e.g., Bushee et al. (2018)). We discuss this alternative specification further in Section 6.1.

We note that an alternative disutility function is $c_{i}\left(r_{i}-E\left[\theta_{i} \mid \Omega_{i}\right]-\eta_{i}\right)^{2} / 2$, whereby manager $i$ receives disutility when she departs from her beliefs of $\theta_{i}$ given her information set at the time of reporting, denoted by $\Omega_{i}$. This alternative specification would not qualitatively or quantitatively affect our results concerning the managers' equilibrium biasing behavior and market pricing. ${ }^{10,11}$

The personal cost in equation (1) captures the essence that managers benefit from more information, endure disutility from distortion, and the manager's report is not fully revealing of her private information. Each manager's objective is to maximize the expected price after disclosure by both firms net of the disutility (1), conditional on her information set. Under the sequential regime, the manager that reports first, denoted as manager 1 , this is given as:

$$
\max _{r_{1}} \mathbb{E}\left[P_{1}-\frac{c_{1}\left(r_{1}-\theta_{1}-\eta_{1}\right)^{2}}{2} \mid \Omega_{1}\right]
$$

where $\Omega_{1}$ denotes manager 1's information set, which includes $s_{1}, \eta_{1}$, and her conjecture of the influence of her report on the second manager's report. Similarly, the manager that reports second, denoted as manager 2 , has the following objective function:

$$
\max _{r_{2}} \mathbb{E}\left[P_{2}-\frac{c_{2}\left(r_{2}-\theta_{2}-\eta_{2}\right)^{2}}{2} \mid \Omega_{2}\right]
$$

where her information set $\Omega_{2}$ consists of $s_{2}, \eta_{2}, r_{1}$, and manager 2's conjecture of manager 1 's reporting strategy. The timeline of the sequential regime is presented in Figure 1 . We allow firms to be heterogeneous in all parameters, i.e., we allow $\tau_{1}^{\theta} \neq \tau_{2}^{\theta}, \tau_{1}^{\varepsilon} \neq \tau_{2}^{\varepsilon}, c_{1} \neq c_{2}$, and $\tau_{1}^{\eta} \neq \tau_{2}^{\eta}$.

\footnotetext{
${ }^{10}$ The results concerning timing preferences in Section 3 would be affected, as this disutility function removes the additional ex-ante utility of the follower due to the informational advantage.

${ }^{11}$ In some prior models of biased reporting, the manager's posterior/conditional expectation of firm value is the same as the realization of her private signal. Consequently, in these settings, considering a cost function that depends on the deviation of the report from the manager's private signal is equivalent to our specification. However, in our setting, the manager's conditional expectation at the time of reporting is not just the realization of the private signal. In particular, the second manager's posterior is determined by both her private signal $s_{2}$ as well as the first manager's report $r_{1}$. Hence, such a utility function does not fit our model.
} 


\section{Equilibrium}

We first analyze the equilibrium of the sequential setting and make comparisons to the simultaneous reporting regime. We then consider timing preferences later in this section.

\section{Reporting strategies: Sequential regime}

We begin by deriving the optimal reporting strategies of the managers. In line with the extant literature (e.g., Stein (1989), Fischer and Verrecchia (2000), Heinle and Verrecchia (2016)), we focus on linear equilibria, where prices are linear in the observed reports. To this end, we conjecture (and later prove) an equilibrium pricing structure where prices are linear in reports:

$$
\left(\begin{array}{l}
P_{1} \\
P_{2}
\end{array}\right)=\left(\begin{array}{ll}
A_{11} & A_{12} \\
A_{21} & A_{22}
\end{array}\right)\left(\begin{array}{l}
r_{1} \\
r_{2}
\end{array}\right)+\left(\begin{array}{l}
Z_{1} \\
Z_{2}
\end{array}\right) .
$$

As shown above, due to the correlation in firm values, the market factors information from both reports when pricing each firm. The terms $A_{11}, A_{12}, A_{21}$, and $A_{22}$ represent the market's weights on the reports $r_{1}$ and $r_{2}$ when pricing the firms, while $Z_{1}$ and $Z_{2}$ are constants. We often refer to the weights $A_{11}, \ldots, A_{22}$ as the market's price response coefficients to the reports $r_{1}$ and $r_{2}$.

Under this conjectured pricing structure, we examine each manager's reporting incentive. Substituting for $P_{2}$ and taking the first-order condition of the second manager's objective function in equation (3) yields

$$
r_{2}=\mathbb{E}\left[\theta_{2} \mid s_{2}, r_{1}\right]+\eta_{2}+\frac{A_{22}}{c_{2}} .
$$

We see that manager 2 extracts information from the first firm's report $r_{1}$ when forming expectations about the fundamental value of her firm, $\theta_{2}$. We focus on linear strategies for manager 2 , whereby the report is linear in $r_{1}$, i.e.,

$$
\frac{\partial \mathbb{E}\left[\theta_{2} \mid r_{1}, r_{2}\right]}{\partial r_{1}}=\frac{\partial r_{2}}{\partial r_{1}}=X
$$

where $X$ is a constant. Following this conjecture, we can derive the optimal report of manager 1 from the first-order condition of the objection function (2):

$$
r_{1}=\mathbb{E}\left[\theta_{1} \mid s_{1}\right]+\eta_{1}+\frac{A_{11}+A_{12} X}{c_{1}}
$$

We see above that the weight $X$ the second manager places on $r_{1}$ also appears in the report 
of the first manager. As we discuss later, this occurs since the market uses both $r_{1}$ and $r_{2}$ when forming beliefs of the value of firm 1 . Additionally, one can see that, since all of the random variables in the model are distributed normally, the report $r_{1}$ is also linear in the signal $s_{1}$ (since $\mathbb{E}\left[\theta_{1} \mid s_{1}\right]$ is linear in $s_{1}$ ) and the bias parameter $\eta_{1}$. Similarly, the second firm's report $r_{2}$ is linear in $s_{2}$ and $\eta_{2}$. The next lemma specifies the weight placed on private information in the reports as well as the weight manager 2 places on $r_{1}$ in her report.

Lemma 1. The managers' reporting strategies are given by

$$
\begin{aligned}
& r_{1}=D_{1} s_{1}+\eta_{1}+\frac{A_{11}+A_{12} X}{c_{1}} \\
& r_{2}=D_{2} s_{2}+X\left(r_{1}-\frac{A_{11}+A_{12} X}{c_{1}}\right)+\eta_{2}+\frac{A_{22}}{c_{2}},
\end{aligned}
$$

$D_{1}$ and $D_{2}$ are strictly positive, while $X$ has the same sign as $\rho$. The coefficients $D_{1}, D_{2}$, and $X$ are given by:

$$
\begin{aligned}
D_{1} & =\frac{\tau_{1}^{\varepsilon}}{\tau_{1}^{\varepsilon}+\tau_{1}^{\theta}}, \\
D_{2} & =\left(\begin{array}{l}
0 \\
1
\end{array}\right)^{T}\left(I+\Sigma \Sigma_{\theta}^{-1}\right)^{-1}\left(\begin{array}{l}
0 \\
1
\end{array}\right), \\
X & =\frac{\tau_{1}^{\varepsilon}+\tau_{1}^{\theta}}{\tau_{1}^{\varepsilon}}\left(\begin{array}{l}
0 \\
1
\end{array}\right)^{T}\left(I+\Sigma \Sigma_{\theta}^{-1}\right)^{-1}\left(\begin{array}{l}
1 \\
0
\end{array}\right),
\end{aligned}
$$

where

$$
\Sigma=\left(\begin{array}{ccc}
\frac{1}{\tau_{1}^{\varepsilon}}+\left(\frac{\tau_{1}^{\varepsilon}+\tau_{1}^{\theta}}{\tau_{1}^{\varepsilon}}\right)^{2} & \frac{1}{\tau_{1}^{\eta}} & 0 \\
0 & & \frac{1}{\tau_{2}^{\varepsilon}}
\end{array}\right)
$$

and $\Sigma_{\theta}$ is the variance-covariance matrix of firms' fundamentals.

We see above that manager 2 extrapolates information from $r_{1}$ when issuing her report. Moreover, both managers consider the price response coefficients $A_{11}, \ldots, A_{22}$ in their reports. The response coefficients $A_{11}, \ldots, A_{22}$ determine the impact of the reports on the prices of the firms and, consequently, they affect the incentives of managers to bias their reports.

\section{Reporting strategies: Simultaneous benchmark}

We proceed by examining the equilibrium strategies in the simultaneous regime where both managers issue their reports at the same time. This provides a benchmark for comparison with the sequential regime and allows us to isolate the forces that arise due to the sequential nature of reporting from the effects driven by correlation between firm fundamentals. We 
denote the price response coefficients in the benchmark case as $A_{11}^{B}, \ldots, A_{22}^{B}$, where $A_{i, j}^{B}$ is the weight on the report of manager $j=1,2$ in the price of firm $i=1,2$ when the reports are issued simultaneously. We denote the weight that the manager of firm $i$ puts on her signal in this scenario as $D_{i}^{B}$, where $i=1,2$. The following lemma summarizes the optimal reporting strategies in the simultaneous benchmark case.

Lemma 2. In the benchmark of simultaneous reporting, manager $i$ 's report is

$$
r_{i}=D_{i}^{B} s_{i}+\eta_{i}+\frac{A_{i i}^{B}}{c_{i}},
$$

where the coefficient $D_{i}^{B}$ is given by

$$
D_{i}^{B}=\frac{\tau_{i}^{\varepsilon}}{\tau_{i}^{\varepsilon}+\tau_{i}^{\theta}},
$$

and $i=1,2$.

When manager 2 reports simultaneously with manager 1 , she does not observe $r_{1}$ and, consequently, she uses only her own signal when determining $r_{2}$. Likewise, manager 1 no longer considers the expected impact of her report on $r_{2}$ when determining $r_{1}$.

Proposition 1. If $\rho \neq 0$ than the following holds true for the coefficients of managers' reports:

$$
\begin{aligned}
& D_{1}=D_{1}^{B} \\
& D_{2}<D_{2}^{B} .
\end{aligned}
$$

If $\rho=0$ then the reporting strategies and the market pricing under the sequential regime and the simultaneous regime are identical.

The weight assigned to the private signal in $r_{1}$ continues to be the same under both regimes for manager 1, as this manager's information set is the same under both regimes. However, manager 2 assigns strictly less weight to her private information in the sequential regime than under the simultaneous regime. The reason is that after observing $r_{1}$, she updates her beliefs concerning her firm's value $\theta_{2}$ and incorporates this information into her report $r_{2}$, which leads to a lower relative weight on her own private signal $s_{2}$. While observing $r_{1}$ may be beneficial for manager 2 , the market in turn receives overall less information from observing both reports in the sequential regime than under the simultaneous regime. This is because of the second manager's lower reliance on her private information $s_{2}$ in the sequential regime. As we show later, this has implications for the efficiency and volatility of prices. 
In what follows, for ease of exposition we focus on the case of positive correlation, i.e., $\rho>0$ (all of the main results also hold for $\rho<0$, unless indicated otherwise).

\section{Manipulation and market beliefs}

We next examine the role of sequentiality on the reporting bias and market pricing. We define the average (or expected) bias that the manager of firm $i$ adds to the report of the firm as $b_{i}=\mathbb{E}\left[r_{i}-\theta_{i}\right]$, so that

$$
\begin{aligned}
& b_{1}=\mathbb{E}\left[\mathbb{E}\left[\theta_{1} \mid s_{1}\right]+\eta_{1}+\frac{A_{11}+A_{12} X}{c_{1}}-\theta_{1}\right]=\frac{A_{11}+A_{12} X}{c_{1}}, \\
& b_{2}=\mathbb{E}\left[\mathbb{E}\left[\theta_{2} \mid s_{2}, r_{1}\right]+\eta_{2}+\frac{A_{22}}{c_{2}}-\theta_{2}\right]=\frac{A_{22}}{c_{2}}
\end{aligned}
$$

We see above that the average bias in the report of the second firm is determined by how much the manager can affect $P_{2}$ through her own report $r_{2}$, i.e., $A_{22}$. In contrast, the average bias in the report by manager 1 includes this incentive as well as the manager's incentive to influence the price of firm 1 indirectly by influencing the report of firm 2 . To derive the optimal biases, we first examine the market's pricing function. The managers' reports can be expressed as:

$$
\begin{aligned}
& r_{1}=D_{1} s_{1}+\eta_{1}+b_{1}, \\
& r_{2}=D_{2} s_{2}+\eta_{2}+X\left(r_{1}-b_{1}\right)+b_{2} .
\end{aligned}
$$

To determine market beliefs of the firm values, we proceed through two steps. First, the market "naively" updates on the managers' private information by filtering the expected biases from the observed reports:

$$
\begin{aligned}
& \tilde{r}_{1}=\frac{r_{1}-b_{1}}{D_{1}}=s_{1}+\frac{1}{D_{1}} \eta_{1}, \\
& \tilde{r}_{2}=\frac{r_{2}-X\left(r_{1}-b_{1}\right)-b_{2}}{D_{2}}=s_{2}+\frac{1}{D_{2}} \eta_{2} .
\end{aligned}
$$

In this way, the market creates unbiased estimates for $\theta_{1}$ and $\theta_{2}$ from the observed reports. As we see later, it is convenient to use the unbiased estimate (or filtered report) $\tilde{r}_{i}$. While the market cannot perfectly infer the manager's signal, a greater weight $D_{i}$ on the manager's private information $s_{i}$ in her report $r_{i}$ allows the market to better extract information regarding $s_{i}$, as the report assigns a higher weight to $s_{i}$ relative to the weight assigned to $\eta_{i}$. In the second step, the market attempts to filter out the signal $s_{i}$ from the unknown component 
$\eta_{i}$. In doing so, the market uses both $r_{1}$ and $r_{2}$, and the manager's reporting strategy, which includes the weights $D_{1}, D_{2}$, and $X$, when trying to infer $s_{i}$. Correspondingly, these weights help to determine the response coefficients $A_{11}, \ldots, A_{22}$ in the market's pricing function, as shown shortly in the next lemma.

Before proceeding, we introduce the following notation in order to characterize the market pricing functions.

Definition 1. Denote

$$
L\left(D_{1}, D_{2}\right)=\left(I+\hat{\Sigma} \Sigma_{\theta}^{-1}\right)^{-1}
$$

where

$$
\hat{\Sigma}=\left(\begin{array}{cc}
\frac{1}{\tau_{1}^{\varepsilon}}+\left(\frac{1}{D_{1}}\right)^{2} \frac{1}{\tau_{1}^{\eta}} & 0 \\
0 & \frac{1}{\tau_{2}^{\varepsilon}}+\left(\frac{1}{D_{2}}\right)^{2} \frac{1}{\tau_{2}^{\eta}}
\end{array}\right)
$$

is the variance-covariance matrix of noise in the reports. We denote the components of $L\left(D_{1}, D_{2}\right)$ by $L_{11}, L_{12}, L_{21}$, and $L_{22}$, which are functions of $D_{1}$ and $D_{2}$.

The components $L_{11}, \ldots, L_{22}$ denote the weights the market puts on the naive updates $\tilde{r}_{i}$ when pricing the firms. In particular,

$$
\left(\begin{array}{l}
P_{1} \\
P_{2}
\end{array}\right)=\left(\begin{array}{l}
\mathbb{E}\left[\theta_{1} \mid \tilde{r}_{1}, \tilde{r}_{2}\right] \\
\mathbb{E}\left[\theta_{2} \mid \tilde{r}_{1}, \tilde{r}_{2}\right]
\end{array}\right)=\left(\begin{array}{cc}
L_{11} & L_{12} \\
L_{21} & L_{22}
\end{array}\right)\left(\begin{array}{l}
\tilde{r}_{1} \\
\tilde{r}_{2}
\end{array}\right) .
$$

Recall that $\tilde{r}_{i}=s_{i}+\frac{\eta_{i}}{D_{i}}=\theta_{i}+\varepsilon_{i}+\frac{\eta_{i}}{D_{i}}$, so the matrix $L$ captures the weights put on the normalized reports about the firm value $\theta_{i}$ with noise $\varepsilon_{i}+\frac{\eta_{i}}{D_{i}}$. The coefficients of matrix $L$ capture the information content behind the reports of the managers.

We can now more easily characterize the price response coefficients as follows.

Lemma 3. The price response coefficients to the reports $r_{1}$ and $r_{2}$ are

$$
\begin{array}{ll}
A_{11}=\frac{L_{11}}{D_{1}}-\frac{L_{12}}{D_{2}} X, & A_{12}=\frac{L_{12}}{D_{2}} \\
A_{21}=\frac{L_{21}}{D_{1}}-\frac{L_{22}}{D_{2}} X, & A_{22}=\frac{L_{22}}{D_{2}},
\end{array}
$$

Moreover, $L_{i j}$ is increasing in $D_{j}$ and decreasing in $D_{-j}$, for $i \in\{1,2\}$ and $j \in\{1,2\}$.

We see above that the price response coefficients are determined by the weights $D_{1}, D_{2}$, and $X$. To see the intuition for Lemma 3 more clearly, recall firm 1's pricing function:

$$
P_{1}=A_{11} r_{1}+A_{12} r_{2}+Z_{1}
$$


The structure of the price coefficients $A_{11}, \ldots, A_{22}$ is more straightforward than it appears. The market first adjusts the report into an unbiased estimator of $\theta_{i}$ by filtering the expected bias and the coefficient on $s_{i}$ (the first step discussed above), and then must infer $\theta_{i}$ (and also $\theta_{-i}$ ) from its conjecture of $s_{i}$ (the second step here). The $L_{i j}$ components signify the second step - these are the market's Bayesian weights assigned to the filtered report $\tilde{r}_{i}$, which it uses to update beliefs regarding $\theta_{i}$ from the conjectured $s_{i}$.

Next, observe that $L_{i j}$ are the weights on the filtered report $\tilde{r}_{j}$, while price response coefficients $A_{i j}$ are the weights on the actual reports $r_{j}$. It follows that each $L_{i j}$ has to be divided by $D_{j}$. Finally, since the report of the second manager contains information from the first manager's report, this redundant information from $r_{1}$ has to be backed out from the report $r_{2}$. For example, the market places a weight of $A_{12}=\frac{L_{12}}{D_{2}}$ on $r_{2}$ when pricing firm 1. However, some of this information is already contained in $r_{1}$. In particular, the second manager incorporates $X \cdot r_{1}$ into her report $r_{2}$. Hence, the market must remove the redundant component, represented by $\frac{L_{12}}{D_{2}} \cdot X$, from the weight they assign to $r_{1}$ when pricing firm 1 . This leads the coefficient to have the structure $A_{11}=\frac{L_{11}}{D_{1}}-\frac{L_{12}}{D_{2}} X$. In sum, the price response coefficients represent the Bayesian weights assigned to the reports to filter out information concerning $\theta_{1}$ and $\theta_{2}$, while also accounting for the overlapping information in the reports.

The second part of Lemma 3 establishes a critical property. We focus the discussion on $L_{11}$, which is the market's Bayesian weight on the filtered report $\tilde{r}_{1}$ when updating beliefs about $\theta_{1}$. Lemma 3 claims that $L_{11}$ is inversely related to the weight $D_{2}$ the second manager places on her private signal $s_{2}$. To see this, note that, as $D_{2}$ increases, the report $r_{2}$ becomes more informative concerning the signal $s_{2}$. This can be seen from the filtered report $\tilde{r}_{2}=s_{2}+\frac{1}{D_{2}} \eta_{2}$; with a higher weight $D_{2}$, the market's conjecture of $s_{2}$ becomes more precise. As a consequence, the filtered signal $\tilde{r}_{1}$ now becomes less important when updating the value of both firms, $\theta_{1}$ and $\theta_{2}$. Accordingly, the market decreases their Bayesian weight on $\tilde{r}_{1}$, which results in the first report $r_{1}$ playing a smaller role in determining market beliefs concerning $\theta_{1}$ and $\theta_{2}$. Hence, the Bayesian weight $L_{11}$ decreases as the second manager's weight on the private signal increases (i.e., $L_{11}$ is decreasing in $D_{2}$ ). ${ }^{12}$ As we see shortly, this property plays a critical role in the results that follow.

Analogous to the sequential regime, the price response coefficients in the benchmark case

\footnotetext{
${ }^{12}$ Likewise, for the same reason as above, the market's Bayesian weight $L_{22}$ on $\tilde{r}_{2}$ when updating beliefs about $\theta_{2}$ increases as $D_{2}$ increases (i.e., $L_{22}$ is positively related to $D_{2}$ )
} 
of simultaneous reporting are similarly derived:

$$
\begin{aligned}
& A_{11}^{B}=\frac{L_{11}^{B}}{D_{1}^{B}}, \quad A_{12}^{B}=\frac{L_{12}^{B}}{D_{2}^{B}} \\
& A_{21}^{B}=\frac{L_{21}^{B}}{D_{1}^{B}}, \quad A_{22}^{B}=\frac{L_{22}^{B}}{D_{2}^{B}} .
\end{aligned}
$$

Note that $X$ does not appear in the coefficients as it equals zero in the simultaneous regime.

We now establish existence and uniqueness of the linear equilibrium in each regime, and compare the managers' manipulation behaviors between the two regimes.

Theorem 1. A unique linear equilibrium exists in both the sequential and simultaneous reporting regimes. In the sequential regime, the bias by manager 1 always exceeds the corresponding bias in the simultaneous regime, i.e.,

$$
b_{1}>b_{1}^{B}
$$

The bias by manager 2 exceeds the corresponding bias in the simultaneous regime if and only if the precision of the second manager's objective function coefficient, $\tau_{2}^{\eta}$, is sufficiently high. That is,

$$
b_{2}>b_{2}^{B} \text { if and only if } \tau_{2}^{\eta}>\bar{\tau}_{2}^{\eta} \text {. }
$$

The first part of Theorem 1 states that the lead (first) manager more heavily biases her report when reports are made sequentially relative to the simultaneous reporting regime. This result is quite strong, as it always holds in our setting without any restriction to fundamentals (other than non-zero correlation) and under heterogeneous firms. To see this, first note that under sequential reporting the follower (second) manager uses both her own private signal and the report of the first manager $r_{1}$ to form beliefs about the value of her firm $\theta_{2}$. Consequently, she assigns a lower weight to her signal than in the simultaneous case and a part of the information is lost to the financial market (i.e., $D_{2}<D_{2}^{B}$ as shown in Proposition 1). This effect captures the information loss of sequential reporting (recall that, in contrast to $r_{2}$, the informativeness of $r_{1}$ is the same under both regimes). The market reacts to this information loss by increasing the weight on the lead manager's report when forming beliefs over both firm values $\theta_{1}$ and $\theta_{2}$ (Lemma 3). In turn, the greater emphasis on $r_{1}$ by the market amplifies the lead manager's incentive to manipulate, leading to a greater 
bias. ${ }^{13}$ In sum, the market gives extra attention to the lead manager's report when firms disclose sequentially, leading the manager to more heavily inflate her report. Additionally, the lead manager's report plays a disproportionate role in determining the market's total information under sequential reporting.

The second part of Theorem 1 establishes that the follower manager also biases more and the market assigns a higher weight to $r_{2}$ if the market's uncertainty about the manager's objective function is sufficiently low; that is, when the market's inference about the second manager's private signal from her report is sufficiently precise (i.e., $\tau_{2}^{\eta}$ is sufficiently high). This is perhaps surprising, as we would not expect the market to place a heavier weight on a less informative report, relative to the simultaneous regime where $r_{2}^{B}$ is more informative.

To see how this emerges, recall from Lemma 1 that the report of the follower in the sequential regime is determined as

$$
r_{2}=D_{2} s_{2}+X\left(r_{1}-\frac{A_{11}+A_{12} X}{c_{1}}\right)+\eta_{2}+\frac{A_{22}}{c_{2}} .
$$

The market is unable to perfectly disentangle the manager's private information $s_{2}$ (with weight $D_{2}$ ) from the noise term $\eta_{2}$. The market's response coefficient on $r_{2}$ captures its inference, as shown by Lemma 3 :

$$
A_{22}=\frac{L_{22}}{D_{2}}
$$

The term $L_{22}$ in the numerator represents the market's Bayesian updating of the manager's private information from the report $r_{2}$, while $D_{2}$ in the denominator is scaling this Bayesian update by the weight the manager places on the signal $s_{2}$ in the report $r_{2}$. Two effects are at play in the market's inference. First, as discussed above, the follower's lower reliance on her private information leads to the loss of information in the report $r_{2}$. This contributes to a less precise inference by the market, resulting in a lower weight under sequentiality than under the simultaneous regime, i.e., $L_{22}<L_{22}^{B}$. However, a second effect is also present, which is

${ }^{13}$ More formally, the average biases in the sequential regime are

$$
\begin{aligned}
& b_{1}=\frac{A_{11}+A_{12} X}{c_{1}}=\frac{L_{11}\left(D_{1}, D_{2}\right)}{c_{1} D_{1}}, \\
& b_{2}=\frac{A_{22}}{c_{2}}=\frac{L_{22}\left(D_{1}, D_{2}\right)}{c_{2} D_{2}},
\end{aligned}
$$

while in the simultaneous case, the biases are determined as

$$
b_{1}^{B}=\frac{L_{11}\left(D_{1}^{B}, D_{2}^{B}\right)}{c_{1} D_{1}^{B}}, \quad b_{2}^{B}=\frac{L_{22}\left(D_{1}^{B}, D_{2}^{B}\right)}{c_{2} D_{2}^{B}} .
$$

Recall that $D_{1}=D_{1}^{B}$ while $D_{2}<D_{2}^{B}$. Consequently, by Lemma $3, L_{11}\left(D_{1}, D_{2}\right)>L_{11}\left(D_{1}^{B}, D_{2}^{B}\right)$, and thus $b_{1}>b_{1}^{B}$. 
related to the first effect. Because of the first effect, the weight that the manager places on her private information, $D_{2}$, is smaller under sequentiality relative to the simultaneous regime, i.e., $D_{2}<D_{2}^{B}$. When updating beliefs about $s_{2}$, the market must filter out this coefficient $D_{2}$, resulting in $L_{22}$ being scaled by $D_{2}$. The effect of information loss $\left(L_{22}<L_{22}^{B}\right)$ decreases the weight $A_{22}$ that the market puts on the report of the follower manager, while the scaling effect $\left(D_{2}<D_{2}^{B}\right)$ increases the weight, relative to the simultaneous regime. If the first effect dominates, the follower biases less than in the simultaneous regime $\left(b_{2}<b_{2}^{B}\right)$, while she biases more if the second effect dominates $\left(b_{2}>b_{2}^{B}\right)$.

We proceed by explaining how the precision of the market's inference of $s_{2}$ from the second report determines which effect dominates. With a high inference from the market (i.e., high $\tau_{2}^{\eta}$ ), the noise in the second report is low and investors can better disentangle $\eta_{2}$ from $D_{2} s_{2}$. The information loss due to sequentiality is also limited when $\tau_{2}^{\eta}$ is high. Indeed, as $\tau_{2}^{\eta} \rightarrow+\infty$, the information loss of the sequential regime as compared to the simultaneous one essentially disappears as the managers' private signals are perfectly inferred by the market in both scenarios. However, the scaling effect is unaffected by the market inference from the second report. The coefficients $D_{2}$ and $D_{2}^{B}$ that determine the weights the follower puts on her private signal capture how much information about $\theta_{2}$ is contained in the report of the second manager as compared to the report of the lead manager. These coefficients do not change in $\tau_{2}^{\eta}$ and the scaling effect does not disappear as $\tau_{2}^{\eta} \rightarrow+\infty$. Consequently, for high $\tau_{2}^{\eta}$, the scaling effect dominates the effect of information loss, resulting in $A_{22}>A_{22}^{B}$ and $b_{2}>b_{2}^{B} \cdot{ }^{14}$ We provide additional discussion regarding this result in Appendix B.

An interesting feature of this equilibrium property is that, as $\tau_{2}^{\eta}$ becomes sufficiently high, $A_{22}$ eventually eclipses $A_{22}^{B}$ precisely because $D_{2}<D_{2}^{B}$. In other words, the lower reliance of information by the second manager under sequentiality, $D_{2}$, causes the market to intensify their extraction of the manager's private information from the report $r_{2}$, relative to the simultaneous regime. Hence, paradoxically, the market's extraction incentive can be amplified in the case where the manager's report is less informative.

Theorem 1 implies that the biasing behavior of firms critically depends on the pattern of reporting. In particular, we expect reporting leaders to exhibit greater manipulation in their reports in industries where reporting is staggered relative to industries where reports are clustered in time. In contrast, follower firms may exhibit greater or lower manipulation

\footnotetext{
${ }^{14}$ Conversely, as $\tau_{2}^{\eta}$ decreases, the second report becomes more noisy and investors cannot easily disentangle $\eta_{2}$ from $D_{2} s_{2}$. While this also occurs under the simultaneous regime, the difference in the information contained in the second report between the two regimes increases in $\tau_{2}^{\eta}$. That is, the information loss of sequential reporting is amplified under low $\tau_{2}^{\eta}$, while the scaling effect remains unaffected. As $\tau_{2}^{\eta}$ drops below the threshold $\bar{\tau}_{2}^{\eta}$, the information loss effect begins to dominate the scaling effect, resulting in $A_{22}<A_{22}^{B}$ and $b_{2}<b_{2}^{B}$.
} 
under staggered reporting, depending on the markets ability to infer the manager's private information from the report. These results also have implications for price efficiency, as summarized in the following proposition.

Proposition 2. The sequential regime entails a greater conditional variance of firm values and lower price volatility relative to the simultaneous regime:

$$
\begin{aligned}
\operatorname{Var}\left[\theta_{i} \mid P_{1}, P_{2}\right] & >\operatorname{Var}^{B}\left[\theta_{i} \mid P_{1}, P_{2}\right], i=1,2 \\
\operatorname{Var}\left[P_{i}\right] & <\operatorname{Var}^{B}\left[P_{i}\right], i=1,2 .
\end{aligned}
$$

Proposition 2 establishes that the posterior variance of firm values in the market's belief is higher if firms report sequentially rather than simultaneously. This follows from the market's loss of information arising from sequentiality, and translates to greater uncertainty regarding the underlying firm values. Proposition 2 additionally shows that, while prices are less efficient, they are also less volatile on average under sequential reporting. This is due to the fact that the information loss regarding $\theta_{2}$ is relevant to the value of both firms, as firm values are correlated. Hence, market beliefs regarding $\theta_{1}$ and $\theta_{2}$ diverge less from the unconditional mean, as beliefs become less sensitive to the reports. We discuss implications and connections to the corresponding empirical literature further in Section 5.

\section{Timing preferences}

We now consider firm preferences over the timing and order of reporting. We first examine the lead manager's ex ante expected payoff under the sequential regime:

$$
\mathbb{E}\left[U_{1}\right]=-\frac{c_{1}}{2}\left(b_{1}^{2}+\mathbb{V a r}\left[\theta_{1} \mid s_{1}\right]\right)
$$

(A formal derivation is included in the Appendix.) The first term within parentheses represents the manager's expected cost of manipulation, captured by $b_{1}^{2}$. The second term is the conditional variance of firm value given the manager's information. ${ }^{15}$ This represents the disutility the manager absorbs from having imperfect information of her firm. Finally, as we have assumed (without loss of generality) that values are mean zero, the ex ante expectation of the price is zero. ${ }^{16}$

As shown in the previous section, the lead manager biases more under the sequential regime. However, the market anticipates the higher bias and adjusts its inference of the

\footnotetext{
${ }^{15}$ Due to normality, the conditional variance is independent of the value of the realized signal, and thus we can express it generically in the ex ante expected utility.

${ }^{16}$ If the expected values were not zero, a constant term equal to expected firm value would need to be added to the right-hand side of equation (6).
} 
report $r_{1}$ by attempting to filter out the higher bias $b_{1}$. In turn, the lead manager endures a greater cost to manipulation without deriving any benefit in terms of the expected price. Moreover, the first manager only observes her own signal in the sequential regime and does not benefit from information spillovers. Consequently, being first only leads to a higher expected manipulation cost without any improvement in accuracy of the report. Hence, the lead manager strictly prefers the simultaneous regime as she can save on manipulation costs and enjoy a strictly higher expected utility.

We can similarly express the follower manager's ex ante expected utility under the sequential regime as above:

$$
\mathbb{E}\left[U_{2}\right]=-\frac{c_{2}}{2}\left(b_{2}^{2}+\mathbb{V a r}\left[\theta_{2} \mid s_{2}, r_{1}\right]\right)
$$

In contrast to the leader, the follower derives informational rents in the sequential regime. The second manager can issue a more precise report after learning more about her fundamental value from the first manager's report. This increase in accuracy is reflected in the conditional variance $\operatorname{Var}\left[\theta_{2} \mid s_{2}, r_{1}\right]$, which is strictly lower than $\operatorname{Var}\left[\theta_{2} \mid s_{2}\right]$. While the follower always benefits from an informational advantage, she may incur a higher or lower manipulation cost. As shown in Theorem 1, the follower incurs a higher biasing cost under the sequential regime when the uncertainty about the manager's objective is sufficiently low (i.e., when the market's inference of $s_{2}$ from $r_{2}$ is sufficiently precise). Under simultaneous reporting, the follower may endure a lower cost from manipulation, but she also loses informational rents. As a result, it is not apparent which reporting regime the follower prefers due to these countervailing forces. This issue is analyzed in the following lemma.

Lemma 4. The second firm prefers simultaneous reporting when it provides a sufficient decrease in the manipulation costs as compared to the loss of informational advantage, formally, when $\tau_{2}^{\eta}>\bar{\tau}_{2}^{\eta}$ and $c_{2}<\bar{c}_{2} \cdot{ }^{17}$

The follower prefers to forgo the informational benefit of observing the leader's report when her cost of manipulation is high enough under sequentiality, captured via two conditions. The first condition is that the market's inference of $s_{2}$ from the report is sufficiently high, $\tau_{2}^{\eta}>\bar{\tau}_{2}^{\eta}$. This follows from Theorem 1, as the follower exhibits a greater bias under sequentiality relative to simultaneous reporting when $\tau_{2}^{\eta}$ is sufficiently high. The second condition is that the follower firm's cost of biasing coefficient, $c_{2}$, is sufficiently low. This is somewhat counter-intuitive, as we would expect the manager to be less concerned with manipulation costs when $c_{2}$ is low. However, under a lower cost, the manager is naturally

\footnotetext{
${ }^{17}$ See the Appendix for the characterization of $\bar{\tau}_{2}^{\eta}$ and $\bar{c}_{2}$.
} 
more inclined to manipulate, and this can lead to a larger, and thus more costly, total biasing cost when $c_{2}$ is low.

As discussed previously, a manager that moves first in the sequential regime always prefers simultaneous reporting. Moreover, as shown in Lemma 4, a manager that moves second prefers simultaneous reporting when the cost of manipulation under sequentiality is sufficiently high. This allows us to identify conditions under which both managers prefer simultaneous reporting to the sequential regime.

Theorem 2. Both managers prefer simultaneous reporting when $\tau_{1}^{\eta}>\bar{\tau}_{1}^{\eta}, c_{1}<\bar{c}_{1}, \tau_{2}^{\eta}>\bar{\tau}_{2}^{\eta}$ and $c_{2}<\bar{c}_{2}$.

Theorem 2 above specifies conditions under which clustered reporting can emerge as an equilibrium when firms can choose the timing of their reports. Under low costs of manipulation, $c_{i}$, and when the market's inference of reports is sufficiently precise, firms prefer to issue reports in tandem with their industry peers. The empirical literature has found evidence consistent with firms strategically timing the release of their forecasts and announcements (e.g., Gong et al. (2019)). Our results may help to reconcile conflicting findings in the literature which have documented both staggered reporting (e.g., Truong (2019)) and clustered reporting (e.g., Tse and Tucker (2010)). Our results also imply cross-industry empirical predictions concerning the reporting patterns of firms (discussed further in the Section 5).

\section{Equilibrium properties and comparative statics}

In this section, we explore a few key equilibrium properties of the model that provide new insights regarding the behavior of firms under sequential reporting. In the following section, we discuss the empirical implications that arise from these results as well as those presented in Section 3. We note that all of the comparative statics with respect to $\rho>0$ that follow hold identically with respect to $|\rho|$, unless indicated otherwise.

\section{Properties of manipulation}

We first examine properties of the managers' biases in the reports. A central assumption of the model is that firm values are correlated, giving rise to informational spillovers. Under the simultaneous regime, stronger correlation reduces the biases of both firms. Greater correlation in values implies that the report from an individual firm becomes less important in pricing this firm because the report of the other firm becomes more informative. This decreases the incentive of each manager to bias the report under simultaneous reporting. We 
note that this effect has been established previously by studies that consider simultaneous reporting (e.g., Strobl (2013), Heinle and Verrecchia (2016)).

However, the presence of sequentiality in reporting introduces an additional effect which can lead to the opposite result. As discussed in Section 3, the market relies on the first manager's report more due to the information loss from sequential reporting, which increases the lead manager's incentive to bias. As the correlation in values increases, this information loss is intensified as the second manager relies on her own private signal even less. In turn, the market places even greater weight on the first report and, as a result, the lead manager's incentive to misreport is amplified. Consequently, manipulation by the lead firm can increase under greater informational spillovers. We note that this property is a novel insight of sequentiality that is in contrast with the extant literature on simultaneous reporting.

Proposition 3. In the simultaneous reporting regime, the bias in firms' reports is decreasing in the correlation $\rho$ :

$$
\frac{d b_{1}^{B}}{d \rho}<0, \quad \frac{d b_{2}^{B}}{d \rho}<0 .
$$

Under sequential reporting, the bias of the second (follower) firm decreases in the correlation:

$$
\frac{d b_{2}}{d \rho}<0
$$

In contrast, the bias in the report of the first (lead) firm increases in the correlation:

$$
\frac{d b_{1}}{d \rho}>0
$$

when the correlation is sufficiently high, i.e. $\rho>T^{\rho}$, the precision of the signal and the precision of the objective function of the first manager are sufficiently high, i.e. $\tau_{1}^{\varepsilon}>T_{1}^{\varepsilon}, \tau_{1}^{\eta}>$ $T_{1}^{\eta}$, and the precision of the signal and the precision of the objective function of the second manager are sufficiently low, i.e. $\tau_{2}^{\varepsilon}<T_{2}^{\varepsilon}, \tau_{2}^{\eta}<T_{2}^{\eta}$. Otherwise, the bias of the first firm decreases in $\rho$.

To better understand the conditions under which the bias increases in the correlation $\rho$, we decompose the disparate effects:

$$
\frac{d b_{1}}{d \rho}=\underbrace{\frac{\partial b_{1}}{\partial \rho}}_{<0}+\underbrace{\frac{\partial b_{1}}{\partial D_{2}} \frac{\partial D_{2}}{\partial \rho}}_{<0} .
$$

The first term on the right-hand side represents the first effect above of additional information present in both reports, which decreases the bias. The next two terms capture the second 
effect of manager 2's lower reliance on $s_{2}$, which increases the bias. The second effect dominates when the follower manager has a stronger incentive to learn from the report of the lead firm. This occurs when there is a sufficiently high informational gain: the follower manager's private information must be sufficiently imprecise (i.e., low $\tau_{2}^{\varepsilon}$ ), the report of the lead manager is sufficiently informative (i.e., high $\tau_{1}^{\eta}$ and $\tau_{1}^{\varepsilon}$ ), and the correlation between firms' fundamentals $\rho$ is sufficiently high. This leads the follower manager to more heavily rely on the leader's report, resulting in a greater informational loss and a larger weight on the first report $r_{1}$. Additionally, for investors to put more weight on the report of the first manager, the noise in the second manager's report should be sufficiently high (i.e., low $\tau_{2}^{\eta}$ ). Proposition 3 provides predictions regarding variation in the biasing behavior of firms across industries where staggered reporting is more prevalent (discussed further in the next section).

We next consider the relative manipulation levels among firms under the sequential regime. In order to provide an analytical result, we impose the additional assumption that firms are symmetric in model primitives. Hence, the predictions that emerge from the following proposition are applicable to more homogeneous industries where sequential reporting is prevalent. We find that the bias levels among firms can be ranked according to the uncertainty about the manager's objective, $\tau^{\eta}$ :

Proposition 4. Assume firms are symmetric (i.e., $c_{1}=c_{2} \equiv c, \tau_{1}^{\eta}=\tau_{2}^{\eta} \equiv \tau^{\eta}, \tau_{1}^{\theta}=$ $\left.\tau_{2}^{\theta} \equiv \tau^{\theta}, \tau_{1}^{\varepsilon}=\tau_{2}^{\varepsilon} \equiv \tau^{\varepsilon}\right)$. Let the average biases of the first and second firm be $b_{1}$ and $b_{2}$, respectively, and let $b^{B}$ be the average bias of each firm under simultaneous reporting. There exist thresholds $\tau_{I}^{\eta}$ and $\tau_{I I}^{\eta}>\tau_{I}^{\eta}$ such that

$$
\begin{cases}b_{2}<b^{B}, & \text { if } \tau^{\eta}<\tau_{I}^{\eta}, \\ b_{2} \in\left[b^{B}, b_{1}\right], & \text { if } \tau^{\eta} \in\left[\tau_{I}^{\eta}, \tau_{I I}^{\eta}\right], \\ b_{2}>b_{1}, & \text { if } \tau^{\eta}>\tau_{I I}^{\eta} .\end{cases}
$$

We see above that the follower's manipulation level is greater than the lead manager's bias when there is lower uncertainty of the manager's objective function. This follows from a similar reasoning as in Theorem 1; with lower uncertainty regarding $\eta_{2}$, the market's inference of $s_{2}$ is very precise, and hence the market places a larger weight on the second manager's report in an attempt to extract the information from $r_{2}$. We see in Proposition 4 that this effect can be so large that it leads to a manipulation level by the follower that exceeds the manipulation level of the first manager. This is perhaps surprising, as the market places a relatively larger weight on the less informative report.

Finally, we examine how the bias levels, $b_{1}$ and $b_{2}$, change in the other parameters of the model. For this analysis, we return to the general case of heterogeneous firms. The results 
are summarized as follows:

Proposition 5. The comparative statics of the manipulation levels $b_{1}$ and $b_{2}$ with respect to the precision parameters of the model are summarized in the following table:

\begin{tabular}{|c|c|c|}
\hline & $\tau_{1}^{\eta}, \tau_{1}^{\varepsilon}, \tau_{2}^{\theta}$ & $\tau_{2}^{\eta}, \tau_{2}^{\varepsilon}, \tau_{1}^{\theta}$ \\
\hline$b_{1}$ & monotonically increasing & monotonically decreasing \\
\hline$b_{2}$ & monotonically decreasing & monotonically increasing \\
\hline
\end{tabular}

These comparative statics also hold for the biases $b_{1}^{B}$ and $b_{2}^{B}$ in the simultaneous regime.

Proposition 5 above shows how a firm's level of manipulation is affected by the characteristics of its peers. In particular, heterogeneity in firm features has a first-order effect on the variation in manipulation levels across industries. This allows for predictions concerning how peer characteristics influence firm misreporting behavior. We see that the lead firm's manipulation $b_{1}$ is increasing in the informativeness of her own report. As $\tau_{1}^{\varepsilon}$ or $\tau_{1}^{\eta}$ increase, the market places greater weight on $r_{1}$, thus increasing the manager's manipulation incentive. Likewise, as the second report becomes relatively more informative through increases in either $\tau_{2}^{\varepsilon}$ or $\tau_{2}^{\eta}$, the market shifts attention away from the leader to the follower, resulting in a lower incentive to bias for the leader. Interestingly, we observe that an increase in the ex ante precision $\tau_{1}^{\theta}$ reduces the lead manager's manipulation. This occurs because the lead manager's report $r_{1}$ becomes less useful for the market as the prior information becomes more precise, leading the market to again shift its attention more towards the second manager. The changes in the follower manager's bias $b_{2}$ are analogous to those of the lead manager. We note that the same properties emerge in the case of simultaneous reporting.

\section{Properties of reporting strategies}

We next consider properties of the equilibrium reporting strategies in the sequential regime. In particular, we study the properties of the weights that each firm manager puts on her private signal and on the report of the other firm.

Proposition 6. The managers' reports have the following properties:

(i) The weight $D_{1}$ of the first manager's signal in her report increases in $\tau_{1}^{\varepsilon}$, and decreases in $\tau_{1}^{\theta}$.

(ii) The weight $X$ of the first manager's report in the report of the second manager increases in $\tau_{1}^{\varepsilon}$ and $\rho$, and decreases in $\tau_{2}^{\varepsilon}{ }^{18}$

\footnotetext{
${ }^{18}$ When $\rho<0$, the weight $X$ is negative and it decreases in $\tau_{1}^{\varepsilon}$, and increases in $\tau_{2}^{\varepsilon}$ and $\rho$ for the same reasons.
} 
(iii) The weight $D_{2}$ of the second manager's signal in her report increases in $\tau_{2}^{\varepsilon}$ and $\tau_{1}^{\theta}$, and decreases in $\tau_{2}^{\theta}, \tau_{1}^{\varepsilon}$ and $\rho$.

First, the weight $D_{i}$ of manager $i$ 's private signal $s_{i}$ in her report is higher if the signal is more precise and is lower if the prior information about the fundamental value $\theta_{i}$ is more precise. In other words, the weight $D_{i}$, and thus the informativeness of the report, increases in the precision $\tau_{i}^{\varepsilon}$ of manager $i$ 's own signal and decreases in the prior precision $\tau_{i}^{\theta}$.

Second, the weight $X$ of the first manager's report $r_{1}$ in the report of the second manager $r_{2}$ increases in the precision of the lead manager's signal $\tau_{1}^{\varepsilon}$ and decreases in the precision of the second manager's signal $\tau_{1}^{\varepsilon}$. As the signal of the first manager becomes more informative, the second manager uses it to a greater extent. Moreover, the first manager also puts a higher weight on the signal in his own report. It follows that the report $r_{1}$ provides more information about $\theta_{1}$ and, consequently, $\theta_{2}$. The second manager relies more on this report and less on her own signal $s_{2}$ when issuing the report $r_{2}$. Conversely, when the precision $\tau_{2}^{\varepsilon}$ of the signal of the second manager increases, the manager decreases the weight $X$ she puts on the report of the first manager.

Third, a greater correlation in firms' fundamentals (higher $\rho$ ) implies that the report of the first manager becomes more informative about the value $\theta_{2}$ of the second firm. The second manager then optimally increases the weight $X$ she puts on the report of the first manager and decreases the weight $D_{2}$ she puts on her own signal.

Finally, the weight $D_{2}$ of the second manager's signal in her report increases in the prior

precision $\tau_{1}^{\theta}$ about the first firm's value. If the prior is more precise, the covariance in the firms' values is lower. Moreover, the first manager's report assigns a lower weight to her private signal $s_{1}$. Overall, the first report $r_{1}$ is less informative about the second firm's value and, consequently, $D_{2}$ is higher.

\section{Properties of price response coefficients}

We now examine the price response coefficients in sequential reporting relative to the simultaneous regime. Recall that the pricing functions in the unique linear equilibrium under the sequential regime are derived as

$$
\begin{aligned}
& P_{1}=A_{11} r_{1}+A_{12} r_{2}+Z_{1}, \\
& P_{2}=A_{21} r_{1}+A_{22} r_{2}+Z_{2} .
\end{aligned}
$$

The corresponding price functions in the simultaneous regime are analogous except the coefficients are denoted with superscript $B$ (i.e., $A_{i j}^{B}$ ). 
Proposition 7. In the simultaneous reporting regime, the price response coefficients decrease in the correlation:

$$
\frac{d A_{11}^{B}}{d \rho}<0, \quad \frac{d A_{22}^{B}}{d \rho}<0 .
$$

If the firms report sequentially, this is in general true only for the second firm:

$$
\frac{d A_{22}}{d \rho}<0 .
$$

In contrast, the price response coefficient of the first firm increases in the correlation:

$$
\frac{d A_{11}}{d \rho}>0,
$$

when $\rho>K^{\rho}, \tau_{1}^{\varepsilon}>K_{1}^{\varepsilon}, \tau_{1}^{\eta}>K_{1}^{\eta}$, and $\tau_{2}^{\varepsilon}<K_{2}^{\varepsilon}$. Otherwise, the price response coefficient of the first firm decreases in $\rho$.

The intuition here is similar to that of Proposition 3, which considers the change in manipulation levels with respect to changes in $\rho$. However, there are additional effects for the first firm in the sequential regime. Since some of the information from $r_{1}$ will appear in $r_{2}$, the market must adjust the coefficient $A_{11}$ so as not to "double count" the information in $r_{1}$. Nevertheless, the lead manager still maintains the stronger incentive to misreport under sequentiality, as she still aims to influence perception of her firm indirectly through the second report $r_{2}$ as well.

In Theorem 1, we saw that the bias by the lead manager is always greater under sequential reporting than under simultaneity. However, the coefficient on $r_{1}$ in $P_{1}$ in the sequential regime, $A_{11}$, can be lower than in the analogous simultaneous regime, $A_{11}^{B}$. In the following proposition, we characterize the relative levels of the coefficients between the two cases:

Proposition 8. The coefficient on $r_{1}$ in $P_{1}$ is greater under the sequential regime,

$$
A_{11}>A_{11}^{B}
$$

if and only if $\tau_{1}^{\eta}>N_{1}^{\eta}, \tau_{1}^{\theta}<N_{1}^{\theta}$, and $\rho>N^{\rho}$. In this case, we additionally have that $A_{21}>A_{21}^{B}$. Similarly, the coefficient on $r_{2}$ in $P_{2}$ is greater under the sequential regime,

$$
A_{22}>A_{22}^{B}
$$

if and only if $\tau_{2}^{\eta}>\bar{\tau}_{2}^{\eta}$. We additionally have that $A_{21}>A_{21}^{B}$ when this condition is satisfied.

It is somewhat counter-intuitive that $A_{11}$, the coefficient under sequentiality, can be lower 
than $A_{11}^{B}$, even though $b_{1}>b_{1}^{B}$. The reason is that, while the market overall relies on the lead manager's report $r_{1}$ more heavily in the sequential regime, some of this reliance occurs indirectly through $r_{2}$. Under sequential reporting, the manager of the second firm uses her own signal and the report of the first firm to form beliefs. Consequently, the market puts less weight on the report of the first firm, because the signal of the first firm is already contained in the report of the second firm. We see that $A_{11}>A_{11}^{B}$ when the information loss in $r_{2}$ is sufficiently high in the sequential case so that the relative informativeness of $r_{1}$ is much higher than the informativeness of $r_{2}$. This holds when fundamentals are highly correlated $\left(\rho>N^{\rho}\right)$, the market's inference of $s_{1}$ from $r_{1}$ is high $\left(\tau_{1}^{\eta}>N_{1}^{\eta}\right)$, and there is greater information asymmetry regarding the lead firm $\left(\tau_{1}^{\theta}<N_{1}^{\theta}\right)$. In contrast, for the follower firm, the coefficient under sequentiality $A_{22}$ is larger than in simultaneous reporting under precisely the same condition $\left(\tau_{2}^{\eta}>\bar{\tau}_{2}^{\eta}\right)$ in which the second manager's bias is larger, $b_{1}>b_{1}^{B}$. As such, the reasoning follows closely to that of Theorem 1 .

Finally, we examine the interim pricing that occurs immediately after the lead manager reports.

Proposition 9. Let $P_{1}^{0}$ and $P_{2}^{0}$ denote the prices at which risk-neutral investors price the firms after only the report of the first firm is issued. Then

$$
\left(\begin{array}{l}
P_{1}^{0} \\
P_{2}^{0}
\end{array}\right)=\left(\begin{array}{l}
\mathbb{E}\left[\theta_{1} \mid r_{1}\right] \\
\mathbb{E}\left[\theta_{2} \mid r_{1}\right]
\end{array}\right)=\left(\begin{array}{l}
A_{1}^{0} r_{1}+Z_{1}^{0} \\
A_{2}^{0} r_{1}+Z_{2}^{0}
\end{array}\right),
$$

where

$$
A_{1}^{0}>A_{11} ; A_{1}^{0}>A_{11}^{B}
$$

If firms are symmetric then

$$
A_{1}^{0}>A_{22}
$$

so that the immediate price response is higher for the firm that issues its report first.

We see that the immediate price response to the report of the first firm, $A_{1}^{0}$, exceeds $A_{11}$ in the sequential regime as well as $A_{11}^{B}$ under simultaneous reporting. This is natural, as before the report of the second firm has been made, the market has less information and hence assigns a higher weight to $r_{1}$ when updating beliefs. Likewise, in the case of symmetric (homogeneous) firms, the immediate price response coefficient of a late announcer (equal to the coefficient $A_{22}$ used in the previous analysis) is lower as compared to the immediate price response coefficient of an early announcer, $A_{1}^{0}$. This is intuitive as the market's Bayesian update is always greater after the first firm's report, corresponding to a greater immediate reaction following $r_{1}$ relative to the reaction following $r_{2}$, when part of the information has 
already been priced. We examine incentives when managers care about the interim price in Section 6.2.

\section{$5 \quad$ Empirical implications}

A sizable empirical literature has investigated the presence of intra-industry information transfers among firms through earnings announcements and managerial forecasts (e.g., Foster (1981), Baginski (1987), Han et al. (1989), Freeman and Tse (1992), Ramnath (2002), Thomas and Zhang (2008), Tse and Tucker (2010), Pandit et al. (2011), Brochet et al. (2018), Gong et al. (2019), Hann et al. (2019), Truong (2019), among others). Our setting captures this important feature and provides several new predictions which, to the best of our knowledge, have hitherto not been explored in the empirical literature. The aim of this section is thus to help guide future empirical investigation; however, we make connections with the corresponding empirical literature where possible.

We should note that for most of our results the timing of reports in our model is determined exogenously, whereas in practice it may sometimes be determined endogenously. As such, there may be some additional properties or reporting characteristics that stem from the endogenous choice of timing, which are in addition to the patterns predicted by our model.

Our first main result (Theorem 1) states that lead firms exhibit greater manipulation in their reports under sequential reporting than in the analogous case of simultaneous reporting. Hence, the results predict that, all else equal, we should observe greater levels of manipulation from lead firms in industries where reports are released in a staggered fashion relative to industries where reports are issued simultaneously. Follower firms similarly exhibit heightened manipulation under sequential reporting relative to the simultaneous regime when the market's inference of the manager's information is more precise (i.e., high $\tau_{2}^{\eta}$ ). This can be the case, for example, in less complex or established industries where investors are able to more easily process firm information releases (e.g., Coles et al. (2008)), whereas the market's inference of reports may be noisier in more complex, high growth, emerging, or rapidly evolving industries. This also implies that in such cases where both lead and follower firms manipulate more, we should see greater overall manipulation within the industry.

Prediction 1. Lead firms (firms that report first) in industries with staggered reporting should exhibit higher levels of manipulation in their reports relative to similar firms in industries with clustered reporting. Follower firms (firms that report later) should exhibit greater manipulation under staggered reporting in industries where the market's inference of infor- 
mation is stronger relative to similar firms in industries with clustered reporting. Total or overall manipulation by firms should be highest among industries with staggered reporting and high market inference.

Recall that, due to the informational rents, the second firm relies less on her private information after observing the report of the first firm in the sequential regime. A number of implications follow from this key equilibrium property. Since some of the follower's private information is "lost" under sequentiality, the reports of late announcers are relatively less informative than early announcers. Relatedly, the report of the lead firm has greater influence in shaping market beliefs. In other words, the market updates more heavily following the report of the lead firm under sequential reporting relative to simultaneous reporting, in which all firm reports are weighed proportionally to the precision of their private information. Finally, due to the information loss, prices are less efficient under sequential reporting for all firms in the industry, including leaders. This occurs because the announcements of follower firms also contain industry information relevant to lead firms.

\section{Prediction 2.}

(i) Early reporters in industries with staggered reporting have greater influence in shaping market beliefs than similar firms in industries with clustered reporting;

(ii) The reports of followers in industries with staggered reporting are less informative relative to similar firms in industries with clustered reporting;

(iii) Prices are less efficient and there is greater ex post information asymmetry for all firms within the industry in industries with staggered reporting, relative to industries with clustered reporting.

(iv) Prices exhibit lower volatility in industries with staggered reporting, relative to industries with clustered reporting.

Some evidence for the above prediction has been documented by Givoly and Palmon (1982), who find that late announcers tend to have less informative reports than early announcing firms. Relatedly, Noh et al. (2020) find that early announcers receive more attention from investors and the media, and generate stronger market reactions, consistent with $(i)$ and $(i i)$ of Prediction 2.

The results also provide implications regarding variation in manipulation across industries. In industries where staggered reporting is more prevalent, we expect manipulation to increase in the strength of informational spillovers captured by $\rho$ (Proposition 3). Likewise, follower firms exhibit a lower bias in industries with higher informational benefits to observing peer reports (i.e., high $\rho$ ), and these effects are stronger in industries where there are 
greater benefits to learning (i.e., when followers have less precise information; Proposition $3)$.

Prediction 3. In industries where reports are staggered and information spillovers are prevalent (i.e., $\rho$ is high enough), the level of manipulation of lead firms should increase in the strength of informational spillovers $(\rho)$ provided that these firms have a strong informational advantage as compared to other firms (i.e., high $\tau_{1}^{\varepsilon}$ and $\tau_{1}^{\eta}$; low $\tau_{2}^{\varepsilon}$ and $\left.\tau_{2}^{\eta}\right)$. In contrast, the level of manipulation of follower firms should always decrease in the strength of information spillovers.

One of the strengths of our setting is that we allow for heterogeneous firms. As such, we are able to provide predictions regarding how firm choices depend on the characteristics of other firms (i.e., variation in firm $i$ 's behavior with changes in the characteristics of firm $j)$. This relates to the recent empirical literature on peer effects in capital markets, which typically examine how the actions of firms are influenced by their industry peers (e.g., Leary and Roberts (2014), Kaustia and Rantala (2015), Grennan (2019), Seo (2020)). To the best of our knowledge, peer effects in misreporting has yet to be investigated in the empirical literature. The predictions we offer below may thus help to guide empirical research in this area.

Proposition 5 demonstrates that a firm's manipulation is greater when the peer firm has a less opaque information environment (high $\tau_{-i}^{\theta}$ ), such as through a higher analyst following of the peer firm. Relatedly, the results imply that firm manipulation is decreasing when the peer has more precise information (high $\tau_{-i}^{\varepsilon}$ ). This can be interpreted, for example, as more precise information release by peer firms in previous periods. Finally, firm manipulation is decreasing when the market's inference of the peer report is stronger (high $\tau_{-i}^{\eta}$ ), which can be the case, for example, when peer firms are less complex or have stronger corporate governance.

Prediction 4. In both reporting regimes, firms (both leaders and followers) exhibit greater manipulation when industry peers have

(i) A less opaque information environment or less information asymmetry between the firm and investors (high $\tau_{-i}^{\theta}$ );

(ii) Less precise information (low $\tau_{-i}^{\varepsilon}$ );

(iii) Lower market inference of reports (low $\left.\tau_{-i}^{\eta}\right)$.

Our results thus allow for sharp predictions regarding how manipulation levels are indirectly affected by peer characteristics. Hence, we provide theoretical underpinnings regarding the different influences on peer behavior, which may be helpful in future empirical investigation on manipulation and peer effects. We note that the above prediction is quite general 
in the sense that it holds irrespective of the reporting pattern (i.e., for both sequential and simultaneous reporting) and holds for both leaders and followers.

The model also offers predictions regarding the relative levels of manipulation between leaders and followers under sequential reporting. We find that leaders tend to exhibit a greater bias than followers when the market's ability to interpret reports is weak, and vice versa when the market's inference of the manager's information from the report is strong (Proposition 4). This prediction is with respect to homogeneous industries where firms share greater similarities in their characteristics (e.g., Parrino (1997)).

Prediction 5. In more homogeneous industries with sequential reporting, early reporters are expected to have a greater bias relative to late reporters when the market's inference of reports is weak, such as in industries with greater complexity or a lower quality of corporate governance. When the market's inference is strong, later reporters should exhibit a greater bias relative to early reporters.

Some evidence for the above prediction has been documented by Gong et al. (2019) and Kim et al. (2019), who find that late announcers exhibit greater manipulation levels in their reports than early announcers. Our results predict that the direction of this relation varies by industry or firm characteristics. While Prediction 5 above is with respect to homogeneous firms, our results provide some guidance regarding the relative bias levels among heterogeneous firms as well. As shown in Proposition 5 of Section 4, changes in the exogenous parameters affect each firm in the sequential regime in opposite ways. For example, an increase in the lead firm's precision $\tau_{1}^{\eta}$ increases $b_{1}$ but decreases $b_{2}$. Proposition 5 can thus help guide comparisons of within-industry relative manipulation levels in heterogeneous industries based on industry features.

We next consider the price response to the reports. The results provide predictions regarding both the immediate market reaction to reports as well to the long-term price association with reports (e.g., Kothari (2001)). In terms of the immediate price reaction, the market reacts more strongly to the report of the lead firm in the sequential regime relative to the reaction in the simultaneous regime. Likewise, in industries where firms are more homogeneous, the immediate market reaction following the leader's report always exceeds the reaction to that of the follower's report when reports are staggered.

Our results also provide implications regarding the long-term price associations with reports. The results imply that in the sequential regime, the long-term impact of the announcement on the price for the lead firm can be greater when the report $r_{1}$ has higher informational spillovers (captured by $\rho$ ). This is in contrast to the simultaneous regime, where the price reactions are decreasing for all firms as informational spillovers increase 
(Proposition 7). Our second set of predictions relates the relative long-term price associations between sequential and simultaneous reporting (Proposition 8). We find that the lead report has a greater long-term price impact in the sequential regime as compared to the simultaneous regime when the market's inference is strong (high $\tau_{1}^{\eta}$ ), the ex ante information asymmetry between the firm and investors is high (low $\tau_{1}^{\theta}$ ), and the correlation is sufficiently high. These testable predictions provide cross-industry variation in the long-term price impact and association of announcements.

Prediction 6. We have the following predictions with respect to the immediate and long-term price association with reports:

(i) In industries which are more homogeneous, the immediate market reaction to early reports is stronger than to later reports when reports are issued sequentially.

(ii) Under sequential reporting, the announcements of lead firms have a greater long-term price impact when informational spillovers are more salient $(\rho)$ and when the industry is less complex or exhibits stronger corporate governance $\left(\tau_{1}^{\eta}\right)$. The reports of follower firms under the sequential regime and all firms in the simultaneous regime exhibit a lower relation with long-term prices as informational spillovers increase.

(iii) Long-term prices exhibit a greater association with reports of lead firms under sequential reporting relative to the simultaneous regime in industries with less complexity or stronger corporate governance $\left(\tau_{1}^{\eta}\right)$, higher information asymmetry $\left(\tau_{1}^{\theta}\right)$, and greater information spillovers $(\rho)$. For follower firms, this is true in less complex industries or industries with stronger corporate governance $\left(\tau_{2}^{\eta}\right)$.

Finally, our results provide implications concerning the pattern in which reports emerge across industries. The presence of strategic timing of announcements and forecasts has been documented in numerous empirical studies, such as Givoly and Palmon (1982), Skinner (1994), Kothari et al. (2009), Tse and Tucker (2010), deHaan et al. (2015), Gong et al. (2019), and Truong (2019). Theorem 2 implies that clustered reporting may be more prevalent in industries where the market can more strongly interpret reports, such as in industries which are less complex, have greater corporate governance, or have a greater concentration of sophisticated or institutional investors. The costs of manipulation must also be sufficiently low, such as in industries where managers have greater discretion over information release or among firms with weaker internal controls or weaker corporate governance. Likewise, more complex industries, where followers typically derive greater informational benefits, may experience more staggered reporting where follower reports are delayed. 
Prediction 7. Firms prefer to report simultaneously and there should be a greater prevalence of clustering in industries with strong market inference of reports and lower manipulation costs for managers.

\section{Extensions}

\subsection{Alternative specification of market uncertainty}

In our baseline model, we assume that the market faces uncertainty about the manager's objective function (i.e., the private information $\eta_{i}$ in equation (1)). This leads the market to imperfectly recover manager $i$ 's private signal $s_{i}$ from her report $r_{i}$. We note that the equilibrium reporting strategies and pricing are quantitatively (and qualitatively) unchanged if, instead of assuming that the market faces uncertainty about the managers' objective functions $\left(\eta_{i}\right)$, we assume that the market observes manager $i$ 's report with noise, as in Versano and Trueman (2017). That is, we assume that the manager issues the report which is a function of her private information $s_{i}$, whereas the market observes or interprets the report with some noise $\eta_{i}$.

For example, investors may have difficulty in processing or interpreting complex financial statements (e.g., Bushee et al. (2018), Chychyla et al. (2019)), which leads the market's inference to be imperfect. We find that this specification is equivalent to the baseline specification of the paper. Moreover, as long as the market uncertainty $\eta_{i}$ is not observed by the manager when the report is issued, the exact form of disutility of the manger can be either of the form $c_{i}\left(r_{i}-\theta_{i}-\eta_{i}\right)^{2} / 2$ (as in equation (1)) or of the form $c_{i}\left(r_{i}-\theta_{i}\right)^{2} / 2$. Both specifications deliver the same qualitative and quantitative results as we currently have in the paper. For brevity, a formal derivation of this alternative setting is not provided, but it is available from the authors upon request.

\subsection{Short-term price considerations}

In the baseline setting, we assume that both managers care about the market price of their firm after the second manager has reported in the sequential regime. We now relax this assumption and allow managers to also be concerned about market beliefs immediately after the first report (i.e., the short-term price) in the sequential regime. We note that this extension directly affects only the reporting strategy of the lead manager. Formally, we 
assume that, under the sequential regime, the lead manager's objective function is given as:

$$
\max _{r_{1}} \mathbb{E}\left[\alpha P_{1}^{0}+(1-\alpha) P_{1}-\frac{c_{1}\left(r_{1}-\theta_{1}-\eta_{1}\right)^{2}}{2} \mid \Omega_{1}\right]
$$

where $\Omega_{1}$ denotes her information set, which includes $s_{1}, \eta_{1}$, and her conjecture of the influence of her report on the second manager's report. The price $P_{1}^{0}=\mathbb{E}\left[\theta_{1} \mid r_{1}\right]$ is set to equal market beliefs immediately after $r_{1}$ is issued, and $\alpha \in[0,1]$ denotes the relative weight manager 1 places on this short-term price. Our baseline model corresponds to the case of $\alpha=0$.

Taking the first-order condition, the reporting strategy of the first manager becomes

$$
r_{1}=\mathbb{E}\left[\theta_{1} \mid s_{1}\right]+\eta_{1}+\alpha \frac{A_{1}^{0}}{c_{1}}+(1-\alpha) \frac{A_{11}+A_{12} X}{c_{1}}
$$

where $A_{1}^{0}$ denotes the short-term price response coefficient for firm 1, as in Section 4 . Note that the informational content of the report does not change relative to the equilibrium of the baseline setting, as the weights the manager puts on $s_{1}$ and $\eta_{1}$ are set according to Bayesian updating and are independent of $\alpha$. The long-term coefficients $A_{11}, \ldots, A_{22}$ and the short-term coefficients $A_{1}^{0}$ and $A_{2}^{0}$ are exactly the same as those defined in Lemma 3 and Proposition 9, respectively. However, short-term considerations influence the first manager's manipulation incentive, leading to the following expected bias:

$$
\begin{aligned}
b_{1} & =\alpha \frac{A_{1}^{0}}{c_{1}}+(1-\alpha) \frac{A_{11}+A_{12} X}{c_{1}} \\
& =\alpha \frac{A_{1}^{0}}{c_{1}}+(1-\alpha) \frac{L_{11}}{c_{1}} \\
& =\alpha \frac{A_{1}^{0}-L_{11} / D_{1}}{c_{1}}+\frac{L_{11}}{D_{1} c_{1}} .
\end{aligned}
$$

We show that the short-term response coefficient is higher than $L_{11} / D_{1}$, and hence the bias is increasing in $\alpha$. Indeed, in the short term the market relies heavily on the report of the lead manager as this is the only source of information. This implies that shortterm incentives only increase the lead manager's incentive to manipulate in the first-period, resulting in a greater bias relative to the baseline setting under sequential reporting. (This bias also always exceeds $b_{1}^{B}$.) Moreover, given that $A_{1}^{0}$ does not depend on $\rho$, the comparative statics of the bias $b_{1}$ with respect to $\rho$ are independent of $\alpha$, and are thus the same as in the baseline model. We summarize these findings in the following proposition. 
Proposition 10. The lead manager's bias $b_{1}$ increases in the weight $\alpha$ on short-term price, and always exceeds the bias of the baseline setting for any $\alpha>0$. The bias $b_{1}$ is higher than in the simultaneous reporting regime, independent of $\alpha$. The comparative statics of $b_{1}$ with respect to correlation $\rho$ are also independent of $\alpha$.

This extension shows that the degree of myopia by the lead manager affects the bias in her report, but does not affect the informativeness of her report, and does not affect the reporting strategy of the second manager. Moreover, the more myopic a manager is, the stronger her preference to report simultaneously over being the lead manager in a sequential reporting regime.

\subsection{Project decisions}

We now extend the baseline setting to examine the presence of managerial decision making over projects. We assume that firm managers must make a project decision after prices are formed at the end of the second period. This extension allows us to consider potential real effects of the information spillovers. The manager of firm $i$ chooses $k_{i}$ to maximize the value of a project opportunity, where the value is given by $I_{i}=-\left(\theta_{i}-k_{i}\right)^{2}$, so that the optimal project decision is

$$
k_{i}=\mathbb{E}\left[\theta_{i} \mid r_{1}, r_{2}, s_{i}\right]
$$

This quadratic specification is meant to be a reduced-form representation for a setting in which a manager can make better or more suitable project decisions when she is more informed of her firm's value $\theta_{i}$. For example, a manager with a better understanding of her firm's operations, product demand, or human capital can more capably choose acquisitions that will optimize synergies. The market prices the investment opportunity as

$$
\begin{aligned}
\mathbb{E}\left[I_{i} \mid r_{1}, r_{2}\right] & =\mathbb{E}\left[-\left(\theta_{i}-\mathbb{E}\left[\theta_{i} \mid r_{1}, r_{2}, s_{i}\right]\right)^{2} \mid r_{1}, r_{2}\right] \\
& =-\mathbb{E}\left[\operatorname{Var}\left[\theta_{i} \mid r_{1}, r_{2}, s_{i}\right]\right]=-\operatorname{Var}\left[\theta_{i} \mid r_{1}, r_{2}, s_{i}\right]
\end{aligned}
$$

Since the reports are normally distributed, the conditional variance does not depend on the realized reports but only on their informational content, and, consequently, the equilibrium reporting strategies are not affected under this extended setting. However, we now have an additional impact on prices and the value of the firm, which depends on whether firms report simultaneously or sequentially, and whether the firm is the first or second to report under the sequential regime. We establish a result that is similar to Proposition 2. 
Corollary 1. The project decision efficiency of the first firm, and thus its expected value, is lower when firms report sequentially than when the firms report simultaneously.

The project decision efficiency of the first firm is lower in the sequential rather than simultaneous reporting environment because of the information loss in the report of second firm, which results in lower overall information available to the first manager at the time of the project decision. This extension of our baseline setting highlights that sequentiality and the resulting information loss can lead to a real efficiency loss in project decisions. Essentially, real decisions, such as projects or investment, become less efficient for industry reporting leaders under sequential reporting, due to the loss of information content in the follower's report. Since this extension does not qualitatively change the analysis and results of our baseline model, we do not provide a formal derivation of the equilibrium of this extension.

\section{Conclusion}

In this study, we consider a parsimonious setting where firms move sequentially and can benefit from information spillovers. We provide comparison to an analogous setting where firms report simultaneously. The model provides a number of results concerning the manipulation incentives of managers, price efficiency and volatility, and price response coefficients. Our results show that the introduction of sequentiality in reporting critically alters the biasing behavior of firms and leads to quite different pricing properties.

A key equilibrium property we find is that the manager who reports second under the sequential regime places lower weight on her private information when issuing her report. Consequently, while the follower has more precise information due to learning, the market's information is now strictly worse relative to the scenario in which there is no learning by either manager (i.e., simultaneous reporting). Due to this information loss, the market places greater weight on the first manager's report. This has two important implications. First, the lead manager manipulates her report more heavily due to the extra attention. Second, the lead manager's report plays an outsized role in determining the market's total information relative to a simultaneous reporting regime. This result is quite general as it always holds in our setting, even as we allow firms to be heterogeneous in all parameters.

Our results provide testable implications. The presence of sequentiality in reports fundamentally affects the reporting behavior of firms. This points to the need for empirical investigation regarding cross-industry manipulation levels. In particular, industries which have reports issued in a staggered pattern should exhibit greater manipulation of their lead 
reporters relative to industries in which reporting is clustered. Additionally, the presence of heterogeneity in firms allows us to provide predictions concerning variation in manipulation levels based on the characteristics of a firm's industry peers. These predictions are also quite general in the sense that they hold for both leaders and followers and hold irrespective of the reporting pattern. 


\section{References}

Aghamolla, C. And I. Guttman (2020): "Strategic timing of IPOs and disclosure: A dynamic model of multiple firms," The Accounting Review, forthcoming.

Alti, A. (2005): "IPO market timing," The Review of Financial Studies, 18, 1105-1138.

BAGinski, S. P. (1987): "Intraindustry information transfers associated with management forecasts of earnings," Journal of Accounting Research, 196-216.

BAnerJeE, A. V. (1992): "A simple model of herd behavior," The quarterly journal of economics, 107, 797-817.

Bertomeu, J., M. Darrough, and W. Xue (2017): "Optimal conservatism with earnings manipulation," Contemporary Accounting Research, 34, 252-284.

BEyER, A. (2009): "Capital market prices, management forecasts, and earnings management," The Accounting Review, 84, 1713-1747.

Beyer, A., I. Guttman, and I. Marinovic (2019): "Earnings management and earnings quality: Theory and evidence," The Accounting Review, 94, 77-101.

Bikhchandani, S., D. Hirshleifer, and I. Welch (1992): "A theory of fads, fashion, custom, and cultural change as informational cascades," Journal of political Economy, 100, 992-1026.

Bratten, B., J. L. Payne, And W. B. Thomas (2016): "Earnings management: Do firms play follow the leader?" Contemporary Accounting Research, 33, 616-643.

Brochet, F., K. Kolev, and A. Lerman (2018): "Information transfer and conference calls," Review of Accounting Studies, 23, 907-957.

Bushee, B. J., I. D. Gow, And D. J. TAYlor (2018): "Linguistic complexity in firm disclosures: Obfuscation or information?" Journal of Accounting Research, 56, 85-121.

Caskey, J., V. Nagar, And P. Petacchi (2010): "Reporting bias with an audit committee," The Accounting Review, 85, 447-481.

Chen, Q., T. Hemmer, And Y. Zhang (2007): "On the relation between conservatism in accounting standards and incentives for earnings management," Journal of Accounting Research, 45, 541-565.

Chychyla, R., A. J. Leone, and M. Minutti-Meza (2019): "Complexity of financial reporting standards and accounting expertise," Journal of Accounting and Economics, 67, 226-253.

Coles, J. L., N. D. Daniel, And L. Naveen (2008): "Boards: Does one size fit all?" Journal of financial economics, 87, 329-356.

DeHaAn, E., T. Shevlin, And J. Thornock (2015): "Market (In)Attention and the Strategic Scheduling and Timing of Earnings Announcements," Journal of Accounting 
and Economics, 60, 36-55.

Dye, R. A. And S. S. Sridhar (2008): "A positive theory of flexibility in accounting standards," Journal of Accounting and Economics, 46, 312-333.

Einhorn, E., N. Langberg, and T. Versano (2018): "Cross-Firm Real Earnings Management," Journal of Accounting Research, 56, 883-911.

Ewert, R. ANd A. Wagenhofer (2005): "Economic effects of tightening accounting standards to restrict earnings management," The Accounting Review, 80, 1101-1124.

Fischer, P. E. And R. E. Verrecchia (2000): "Reporting bias," The Accounting Review, $75,229-245$.

Foster, G. (1981): "Intra-industry information transfers associated with earnings releases," Journal of Accounting and Economics, 3, 201-232.

Freeman, R. And S. Tse (1992): "An earnings prediction approach to examining intercompany information transfers," Journal of Accounting and Economics, 15, 509-523.

FrIedman, H. L. (2014): "Implications of power: When the CEO can pressure the CFO to bias reports," Journal of Accounting and Economics, 58, 117-141.

GaO, P. And G. Zhang (2019): "Accounting Manipulation, Peer Pressure, and Internal Control," The Accounting Review, 94, 127-151.

Givoly, D. And D. Palmon (1982): "Timeliness of Annual Earnings Announcements: Some Empirical Evidence," The Accounting Review, 57, 486-508.

Gong, G., L. Y. Li, AND H. Yin (2019): "Relative performance evaluation and the timing of earnings release," Journal of Accounting and Economics, 67, 358 - 386.

Goodman, T. H., M. Neamtiu, N. Shroff, and H. D. White (2013): "Management forecast quality and capital investment decisions," The Accounting Review, 89, 331-365.

Graham, J. R., C. R. Harvey, and S. Rajgopal (2005): "The economic implications of corporate financial reporting," Journal of accounting and economics, 40, 3-73.

Grennan, J. (2019): "Dividend payments as a response to peer influence," Journal of Financial Economics, 131, 549-570.

Guttman, I., O. Kadan, And E. Kandel (2006): "A rational expectations theory of kinks in financial reporting," The Accounting Review, 81, 811-848.

Han, J. C., J. J. Wild, And K. Ramesh (1989): "Managers' earnings forecasts and intra-industry information transfers," Journal of Accounting and Economics, 11, 3-33.

Hann, R. N., H. Kim, And Y. Zheng (2019): "Intra-industry information transfers: evidence from changes in implied volatility around earnings announcements," Review of Accounting Studies, 24, 927-971.

Heinle, M. S. And R. E. Verrecchia (2016): "Bias and the Commitment to Disclosure," Management Science, 62, 2859-2870. 
Jorgensen, B. N. And M. T. Kirschenheiter (2012): "Interactive discretionary disclosures," Contemporary Accounting Research, 29, 382-397.

Jovanovic, B. (1982): "Truthful disclosure of information," The Bell Journal of Economics, 36-44.

Kaustia, M. And V. Rantala (2015): "Social learning and corporate peer effects," Journal of Financial Economics, 117, 653-669.

Kedia, S., K. Koh, and S. Rajgopal (2015): "Evidence on contagion in earnings management," The Accounting Review, 90, 2337-2373.

Kim, M. P., S. R. Pierce, And I. Yeung (2019): "Eleventh-Hour Earnings Management and Financial Reporting Timeliness," Working paper.

Kirschenheiter, M. And N. D. Melumad (2002): "Can big bath and earnings smoothing co-exist as equilibrium financial reporting strategies?" Journal of Accounting Research, 40, 761-796.

Kothari, S. (2001): "Capital markets research in accounting," Journal of accounting and economics, 31, 105-231.

Kothari, S. P., S. Shu, And P. D. Wysocki (2009): "Do Managers Withhold Bad News?" Journal of Accounting Research, 47, 241-276.

LEARY, M. T. AND M. R. RoBerts (2014): "Do peer firms affect corporate financial policy?" The Journal of Finance, 69, 139-178.

Noh, S., E. C. So, And R. S. Verdi (2020): "Calendar Rotations: A New Approach for Studying the Impact of Timing using Earnings Announcements," Journal of Financial Economics, Forthcoming.

Pandit, S., C. E. Wasley, And T. ZaCh (2011): "Information externalities along the supply chain: The economic determinants of suppliers stock price reaction to their customers earnings announcements," Contemporary Accounting Research, 28, 1304-1343.

PARRINo, R. (1997): "CEO turnover and outside succession a cross-sectional analysis," Journal of financial Economics, 46, 165-197.

Persons, J. C. and V. A. Warther (1997): "Boom and bust patterns in the adoption of financial innovations," The Review of Financial Studies, 10, 939-967.

Ramnath, S. (2002): "Investor and analyst reactions to earnings announcements of related firms: An empirical analysis," Journal of Accounting Research, 40, 1351-1376.

SEO, H. (2020): "Peer effects in corporate disclosure decisions," Journal of Accounting and Economics, 101364.

Skinner, D. J. (1994): "Why Firms Voluntarily Disclose Bad News," Journal of Accounting Research, 32, 38-60.

Stein, J. C. (1989): "Efficient capital markets, inefficient firms: A model of myopic corpo- 
rate behavior," The Quarterly Journal of Economics, 104, 655-669.

Strobl, G. (2013): "Earnings Manipulation and the Cost of Capital," Journal of Accounting Research, 51, 449-473.

Thomas, J. And F. Zhang (2008): "Overreaction to Intra-industry Information Transfers?" Journal of Accounting Research, 46, 909-940.

Trueman, B. (1990): "Theories of earnings-announcement timing," Journal of Accounting and Economics, 13, 285-301.

Trueman, B. And S. Titman (1988): "An explanation for accounting income smoothing," Journal of accounting research, 127-139.

Truong, P. (2019): "The Disciplining Effect of Peers: Evidence from the Timing of Earnings Announcements," Working paper.

Tse, S. And J. W. TuCKer (2010): "Within-industry timing of earnings warnings: do managers herd?" Review of Accounting Studies, 15, 879-914.

VerrecchiA, R. E. (1983): "Discretionary disclosure," Journal of accounting and economics, 5, 179-194.

Versano, T. and B. Trueman (2017): "Expectations management," The Accounting Review, 92, 227-246. 


\section{Appendix}

\section{A Proofs}

\section{A.1 Proof of Lemma 1}

Recall that

$$
\begin{aligned}
& r_{1}=\mathbb{E}\left[\theta_{1} \mid s_{1}\right]+\eta_{1}+\frac{A_{11}+A_{12} X}{c_{1}}, \\
& r_{2}=\mathbb{E}\left[\theta_{2} \mid s_{2}, r_{1}\right]+\eta_{2}+\frac{A_{22}}{c_{2}} .
\end{aligned}
$$

The properties of Bayesian updating based on normally distributed signals allow us to derive the coefficient $D_{1}$ in the reporting strategy of the first manager

$$
\mathbb{E}\left[\theta_{1} \mid s_{1}\right]=\frac{s_{1} \tau_{1}^{\varepsilon}+\mathbb{E}\left[\theta_{1}\right] \tau_{1}^{\theta}}{\tau_{1}^{\varepsilon}+\tau_{1}^{\theta}}=s_{1} \frac{\tau_{1}^{\varepsilon}}{\tau_{1}^{\varepsilon}+\tau_{1}^{\theta}}=D_{1} s_{1}
$$

To compute $\mathbb{E}\left[\theta_{2} \mid s_{2}, r_{1}\right]$, recall that the second manger observes his own signal $s_{2}=\theta_{2}+\varepsilon_{2}$ and the first manager's report $r_{1}$. Observing $r_{1}$ is equivalent to observing

$$
\tilde{r}_{1}=\frac{r_{1}-\frac{A_{11}+A_{12} X}{c_{1}}}{D_{1}}=\theta_{1}+\varepsilon_{1}+\frac{1}{D_{1}} \eta_{1},
$$

Based on the properties of Bayesian updating, we have that

$$
\left(\begin{array}{l}
\mathbb{E}\left[\theta_{1} \mid s_{2}, r_{1}\right] \\
\mathbb{E}\left[\theta_{2} \mid s_{2}, r_{1}\right]
\end{array}\right)=\left(\Sigma^{-1}+\Sigma_{\theta}^{-1}\right)^{-1}\left(\Sigma^{-1}\left(\begin{array}{c}
\tilde{r}_{1} \\
s_{2}
\end{array}\right)+\Sigma_{\theta}^{-1}\left(\begin{array}{l}
\mathbb{E}\left[\theta_{1}\right] \\
\mathbb{E}\left[\theta_{2}\right]
\end{array}\right)\right)
$$

where $\Sigma_{\theta}$ is the prior covariance matrix of fundamental values and

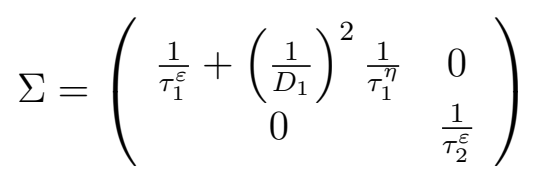

is the covariance matrix of the noise in the signals $\tilde{r}_{1}$ and $s_{2}$. Extracting $\mathbb{E}\left[\theta_{2} \mid s_{2}, r_{1}\right]$ and substituting $D_{1}=\frac{\tau_{1}^{\varepsilon}}{\tau_{1}^{\varepsilon}+\tau_{1}^{\theta}}$, we obtain 


$$
\begin{aligned}
\mathbb{E}\left[\theta_{2} \mid s_{2}, r_{1}\right] & =\left(\begin{array}{l}
0 \\
1
\end{array}\right)^{T}\left(I+\Sigma_{1} \Sigma_{\theta}^{-1}\right)^{-1}\left(\begin{array}{l}
0 \\
1
\end{array}\right) s_{2}+\left(\begin{array}{l}
0 \\
1
\end{array}\right)^{T}\left(I+\Sigma \Sigma_{\theta}^{-1}\right)^{-1}\left(\begin{array}{l}
1 \\
0
\end{array}\right) \tilde{r}_{1} \\
& =\left(\begin{array}{l}
0 \\
1
\end{array}\right)^{T}\left(I+\Sigma_{1} \Sigma_{\theta}^{-1}\right)^{-1}\left(\begin{array}{l}
0 \\
1
\end{array}\right) s_{2}+\left(\begin{array}{l}
0 \\
1
\end{array}\right)^{T}\left(I+\Sigma \Sigma_{\theta}^{-1}\right)^{-1}\left(\begin{array}{l}
1 \\
0
\end{array}\right) \frac{r_{1}-\frac{A_{11}+A_{12} X}{c_{1}}}{D_{1}} \\
& =D_{2} s_{2}+X\left(r_{1}-\frac{A_{11}+A_{12} X}{c_{1}}\right) .
\end{aligned}
$$

\section{A.2 Proof of Lemma 2}

When managers report simultaneously, the second manager does not observe the first manager's report when choosing his own. Consequently, $\frac{\partial r_{2}}{\partial r_{1}}=0$ and the reports are given by

$$
\begin{aligned}
& r_{1}=\mathbb{E}\left[\theta_{1} \mid s_{1}\right]+\eta_{1}+\frac{A_{11}^{B}}{c_{1}}, \\
& r_{2}=\mathbb{E}\left[\theta_{2} \mid s_{2}\right]+\eta_{2}+\frac{A_{22}^{B}}{c_{1}} .
\end{aligned}
$$

Observe that

$$
\mathbb{E}\left[\theta_{i} \mid s_{i}\right]=\frac{s_{i} \tau_{i}^{\varepsilon}+\mathbb{E}\left[\theta_{i}\right] \tau_{i}^{\theta}}{\tau_{i}^{\varepsilon}+\tau_{i}^{\theta}}=s_{i} \frac{\tau_{i}^{\varepsilon}}{\tau_{i}^{\varepsilon}+\tau_{i}^{\theta}}=D_{i}^{B} s_{i}
$$

\section{A.3 Proof of Proposition 1}

When $\rho=0$, the second manager cannot extract any information from the first manager's report, so $X=0$ and the coefficients $D_{2}$ and $D_{2}^{B}$ coincide. Consequently, investors' pricing functions also coincide and two cases are identical.

We compute that

$$
D_{2}^{B}-D_{2}=\rho^{2} L\left(\rho, \tau_{1}^{\varepsilon}, \tau_{2}^{\varepsilon}, \tau_{1}^{\theta}, \tau_{2}^{\theta}, \tau_{1}^{\eta}, \tau_{2}^{\eta}\right)
$$

where $L>0$. It follows that $D_{2}^{B}>D_{2}$ when $\rho \neq 0$.

\section{A.4 Proof of Lemma 3}

Investors observe the reports $r_{1}$ and $r_{2}$, which is equivalent to observing normalized reports introduced in (4)

$$
\begin{aligned}
& \tilde{r}_{1}=\frac{r_{1}-b_{1}}{D_{1}}=s_{1}+\frac{1}{D_{1}} \eta_{1}, \\
& \tilde{r}_{2}=\frac{r_{2}-X\left(r_{1}-b_{1}\right)-b_{2}}{D_{2}}=s_{2}+\frac{1}{D_{2}} \eta_{2} .
\end{aligned}
$$


Investors price the firms based on the information inferred from these reports. Based on the properties of Bayesian updating, we have that

$$
\left(\begin{array}{l}
P_{1} \\
P_{2}
\end{array}\right)=\left(\begin{array}{l}
\mathbb{E}\left[\theta_{1} \mid \tilde{r}_{1}, \tilde{r}_{2}\right] \\
\mathbb{E}\left[\theta_{2} \mid \tilde{r}_{1}, \tilde{r}_{2}\right]
\end{array}\right)=\left(\hat{\Sigma}^{-1}+\Sigma_{\theta}^{-1}\right)^{-1}\left(\hat{\Sigma}^{-1}\left(\begin{array}{c}
\tilde{r}_{1} \\
\tilde{r}_{2}
\end{array}\right)+\Sigma_{\theta}^{-1}\left(\begin{array}{l}
\mathbb{E}\left[\theta_{1}\right] \\
\mathbb{E}\left[\theta_{2}\right]
\end{array}\right)\right),
$$

where

$$
\hat{\Sigma}=\left(\begin{array}{cc}
\frac{1}{\tau_{1}^{\varepsilon}}+\left(\frac{1}{D_{1}}\right)^{2} \frac{1}{\tau_{1}^{\eta}} & 0 \\
0 & \frac{1}{\tau_{2}^{\varepsilon}}+\left(\frac{1}{D_{2}}\right)^{2} \frac{1}{\tau_{2}^{\eta}}
\end{array}\right)
$$

is the variance-covariance matrix of noise in the normalized reports. Given zero prior expectation of the firm values, we further simplify

$$
\left(\begin{array}{l}
P_{1} \\
P_{2}
\end{array}\right)=L\left(\begin{array}{c}
\tilde{r}_{1} \\
\tilde{r}_{2}
\end{array}\right)
$$

where $L\left(D_{1}, D_{2}\right)=\left(I+\hat{\Sigma} \Sigma_{\theta}^{-1}\right)^{-1}$. Substituting normalized reports, we obtain

$$
\begin{aligned}
\left(\begin{array}{l}
P_{1} \\
P_{2}
\end{array}\right) & =L\left(\begin{array}{c}
\frac{r_{1}-b_{1}}{D_{1}} \\
\frac{r_{2}-X\left(r_{1}-b_{1}\right)-b_{2}}{D_{2}}
\end{array}\right)=\left(\begin{array}{c}
\left(\frac{L_{11}}{D_{1}}-\frac{L_{12}}{D_{2}} X\right)\left(r_{1}-b_{1}\right)+\frac{L_{12}}{D_{2}}\left(r_{2}-b_{2}\right) \\
\left.\frac{L_{21}}{D_{1}}-\frac{L_{22}}{D_{2}} X\right)\left(r_{1}-b_{1}\right)+\frac{L_{22}}{D_{2}}\left(r_{2}-b_{2}\right)
\end{array}\right) \\
& =\left(\begin{array}{c}
A_{11}\left(r_{1}-b_{1}\right)+A_{12}\left(r_{2}-b_{2}\right) \\
A_{21}\left(r_{1}-b_{1}\right)+A_{22}\left(r_{2}-b_{2}\right)
\end{array}\right),
\end{aligned}
$$

and indeed the price response coefficients are the ones indicated in the lemma. Computing the signs of the derivatives of the coefficients of the matrix $L$ with respect to $D_{1}$ and $D_{2}$ is straightforward.

\section{A.5 Proof of Theorem 1}

Recall that we conjectured that the report $r_{2}$ is linear the report $r_{1}$ and that prices are linear in reports as well. All the coefficients of the reporting strategies as well as the coefficients in the pricing function were then derived uniquely. Consequently, the linear equilibrium we derived is unique. 
We compute the biases as follows:

$$
\begin{aligned}
& b_{1}=\frac{A_{11}+A_{12} X}{c_{1}}=\frac{L_{11}\left(D_{1}, D_{2}\right)}{c_{1} D_{1}}, \\
& b_{2}=\frac{A_{22}}{c_{2}}=\frac{L_{22}\left(D_{1}, D_{2}\right)}{c_{2} D_{2}} . \\
& b_{1}^{B}=\frac{A_{11}^{B}}{c_{1}}=\frac{L_{11}\left(D_{1}^{B}, D_{2}^{B}\right)}{c_{1} D_{1}^{B}}, \\
& b_{2}^{B}=\frac{A_{22}^{B}}{c_{2}}=\frac{L_{22}\left(D_{1}^{B}, D_{2}^{B}\right)}{c_{2} D_{2}^{B}} .
\end{aligned}
$$

Recall that $D_{1}=D_{1}^{B}$ while $D_{2}<D_{2}^{B}$. Consequently, $L_{11}\left(D_{1}, D_{2}\right)>L_{11}\left(D_{1}^{B}, D_{2}^{B}\right)$ (Lemma 3 shows that $L_{11}$ decreases in the second argument) and $b_{1}>b_{1}^{B}$.

We substitute $D_{i}$ and $D_{i}^{B}, i=1,2$ and show that $b_{2}-b_{2}^{B}$ has the same sign as $M \tau_{2}^{\eta}-N$, where

$$
\begin{aligned}
M & =\left(\left(\tau_{1}^{\theta}\right)^{3}+\left(\tau_{1}^{\varepsilon}\right)^{2}\left(\tau_{1}^{\eta}\left(1-\rho^{2}\right)+\tau_{1}^{\theta}\right)+\tau_{1}^{\varepsilon} \tau_{1}^{\theta}\left(\tau_{1}^{\eta}+2 \tau_{1}^{\theta}\right)\right) \tau_{2}^{\varepsilon} \tau_{2}^{\theta}>0 \\
N & =\tau_{2}^{\theta}\left(\tau_{1}^{\varepsilon}+\tau_{1}^{\theta}\right)\left(\tau_{2}^{\varepsilon}+\tau_{2}^{\theta}\right)\left(\left(\tau_{1}^{\theta}\right)^{2}+\tau_{1}^{\varepsilon}\left(\tau_{1}^{\eta}+\tau_{1}^{\theta}\right)\right)>0 .
\end{aligned}
$$

I follows that $b_{2}>b_{2}^{B}$ if and only if $\tau_{2}^{\eta}>\bar{\tau}_{2}^{\eta}=\frac{N}{M}$.

\section{A.6 Proof of Proposition 2}

Let us first prove that the posterior variances of the firm values conditional on prices are lower in the benchmark case. Observe that for $i=1,2$

$$
\operatorname{Var}\left[\theta_{i} \mid P_{1}, P_{2}\right]=\operatorname{Var}\left[\theta_{i} \mid r_{1}, r_{2}\right]=\mathbb{V a r}\left[\theta_{i} \mid \tilde{r}_{1}, \tilde{r}_{2}\right]
$$

where the normalized reports $\tilde{r}_{1}$ and $\tilde{r}_{2}$ were defined in (4). The posterior variance-covariance of the firm values, conditional on observing the normalized returns, is

$$
\left(\hat{\Sigma}^{-1}\left(D_{1}, D_{2}\right)+\Sigma_{\theta}^{-1}\right)^{-1}
$$

where $\hat{\Sigma}\left(D_{1}, D_{2}\right)$ is the variance-covariance matrix of noise in the normalized reports introduced in Lemma 3.

The posterior variance of the firm $i$ 's value conditional on the prices is then the element $(i, i)$ of the posterior variance-covariance matrix:

$$
\operatorname{Var}\left[\theta_{i} \mid P_{1}, P_{2}\right]=\left.\left(\hat{\Sigma}^{-1}\left(D_{1}, D_{2}\right)+\Sigma_{\theta}^{-1}\right)^{-1}\right|_{i, i}
$$

Similarly, in the benchmark model of simultaneous reporting, the posterior variance of 
the firm $i$ 's value conditional on the prices is

$$
\operatorname{Var}^{B}\left[\theta_{i} \mid P_{1}, P_{2}\right]=\left.\left(\hat{\Sigma}^{-1}\left(D_{1}^{B}, D_{2}^{B}\right)+\Sigma_{\theta}^{-1}\right)^{-1}\right|_{i, i} .
$$

Recall that $D_{1}^{B}=D_{1}$ and $D_{2}^{B}>D_{2}$. The difference in the variances in the sequential and simultaneous reporting cases is then computed to be equal to

$$
\operatorname{Var}\left[\theta_{i} \mid P_{1}, P_{2}\right]-\operatorname{Var}^{B}\left[\theta_{i} \mid P_{1}, P_{2}\right]=C_{i} \rho^{2}\left(D_{2}^{B}-D_{2}\right)
$$

where $C_{i}>0$ is some positive function of parameters of the model.

Now let us prove that the variance of prices is lower in the sequential reporting model as compared to the simultaneous reporting benchmark. Recall from the proof of Lemma 3 that

$$
\left(\begin{array}{l}
P_{1} \\
P_{2}
\end{array}\right)=L\left(\begin{array}{l}
\tilde{r}_{1} \\
\tilde{r}_{2}
\end{array}\right)
$$

where $L\left(D_{1}, D_{2}\right)=\left(I+\hat{\Sigma} \Sigma_{\theta}^{-1}\right)^{-1}$, so that

$$
\begin{aligned}
\operatorname{Var}\left[P_{i}\right]= & \operatorname{Var}\left[L_{i 1} \tilde{r}_{1}+L_{i 2} \tilde{r}_{2}\right] \\
= & L_{i 1}^{2} \operatorname{Var}\left[\tilde{r}_{1}\right]+L_{i 2}^{2} \operatorname{Var}\left[\tilde{r}_{2}\right]+2 L_{i 1} L_{i 2} \operatorname{Cov}\left[\tilde{r}_{1}, \tilde{r}_{2}\right] \\
= & L_{i 1}^{2} \operatorname{Var}\left[s_{1}+\frac{1}{D_{1}} \eta_{1}\right]+L_{i 2}^{2} \mathbb{V} a r\left[s_{2}+\frac{1}{D_{2}} \eta_{2}\right]+2 L_{i 1} L_{i 2} \mathbb{C o v}\left[s_{1}+\frac{1}{D_{1}} \eta_{1}, s_{2}+\frac{1}{D_{2}} \eta_{2}\right] \\
= & L_{i 1}^{2}\left[\frac{1}{\tau_{1}^{\theta}}+\frac{1}{\tau_{1}^{\varepsilon}}+\frac{1}{D_{1}^{2} \tau_{1}^{\eta}}\right]+L_{i 2}^{2}\left[\frac{1}{\tau_{2}^{\theta}}+\frac{1}{\tau_{2}^{\varepsilon}}+\frac{1}{D_{2}^{2} \tau_{2}^{\eta}}\right]+2 L_{i 1} L_{i 2} \frac{\rho}{\sqrt{\tau_{1}^{\theta} \tau_{2}^{\theta}}} \\
= & \left(L_{i 1}\left(D_{1}, D_{2}\right)\right)^{2}\left[\frac{1}{\tau_{1}^{\theta}}+\frac{1}{\tau_{1}^{\varepsilon}}+\frac{1}{D_{1}^{2} \tau_{1}^{\eta}}\right]+\left(L_{i 2}\left(D_{1}, D_{2}\right)\right)^{2}\left[\frac{1}{\tau_{2}^{\theta}}+\frac{1}{\tau_{2}^{\varepsilon}}+\frac{1}{D_{2}^{2} \tau_{2}^{\eta}}\right] \\
& +2 L_{i 1}\left(D_{1}, D_{2}\right) L_{i 2}\left(D_{1}, D_{2}\right) \frac{\rho}{\sqrt{\tau_{1}^{\theta} \tau_{2}^{\theta}}}
\end{aligned}
$$

Similarly, in the benchmark of simultaneous reporting,

$$
\begin{aligned}
\operatorname{Var}^{B}\left[P_{i}\right]= & \left(L_{i 1}\left(D_{1}^{B}, D_{2}^{B}\right)\right)^{2}\left[\frac{1}{\tau_{1}^{\theta}}+\frac{1}{\tau_{1}^{\varepsilon}}+\frac{1}{\left(D_{1}^{B}\right)^{2} \tau_{1}^{\eta}}\right]+\left(L_{i 2}\left(D_{1}^{B}, D_{2}^{B}\right)\right)^{2}\left[\frac{1}{\tau_{2}^{\theta}}+\frac{1}{\tau_{2}^{\varepsilon}}+\frac{1}{\left(D_{2}^{B}\right)^{2} \tau_{2}^{\eta}}\right] \\
& +2 L_{i 1}\left(D_{1}^{B}, D_{2}^{B}\right) L_{i 2}\left(D_{1}^{B}, D_{2}^{B}\right) \frac{\rho}{\sqrt{\tau_{1}^{\theta} \tau_{2}^{\theta}}}
\end{aligned}
$$

Recall that $D_{1}^{B}=D_{1}$ and $D_{2}^{B}>D_{2}$. The difference in the variances of prices in the sequential and simultaneous reporting cases is then computed to be strictly positive if $\rho \neq 0$.

\section{A.7 Derivation of Utilities for Timing Preferences}

We present the following lemma: 
Lemma 5. Ex-ante utilities of managers are given by:

$$
\begin{aligned}
\mathbb{E}\left[U_{1}\right] & =-\frac{c_{1}}{2}\left(b_{1}^{2}+\operatorname{Var}\left[\theta_{1} \mid s_{1}\right]\right), \\
\mathbb{E}\left[U_{1}^{B}\right] & =-\frac{c_{1}}{2}\left(\left(b_{1}^{B}\right)^{2}+\operatorname{Var}\left[\theta_{1} \mid s_{1}\right]\right)>\mathbb{E}\left[U_{1}\right], \\
\mathbb{E}\left[U_{2}\right] & =-\frac{c_{2}}{2}\left(b_{2}^{2}+\operatorname{Var}\left[\theta_{2} \mid s_{2}, r_{1}\right]\right), \\
\mathbb{E}\left[U_{2}^{B}\right] & =-\frac{c_{2}}{2}\left(\left(b_{2}^{B}\right)^{2}+\operatorname{Var}\left[\theta_{2} \mid s_{2}\right]\right) .
\end{aligned}
$$

Proof. Recall that the manager $i$, where $i=1,2$, discloses the report

$$
r_{i}=\mathbb{E}\left[\theta_{i} \mid \Omega_{i}\right]+\eta_{i}+b_{i}
$$

where $\Omega_{1}=\left\{s_{1}\right\}$ and $\Omega_{2}=\left\{s_{2}, r_{1}\right\}$. Substituting this report in the ex-ante utility of the manager $i$ we have

$$
\begin{aligned}
U_{i} & =\mathbb{E}\left[\max _{r_{i}} \mathbb{E}\left[P_{i}-\frac{c_{i}\left(r_{i}-\theta_{i}-\eta_{i}\right)^{2}}{2} \mid \Omega_{i}\right]\right] \\
& =\mathbb{E}\left[P_{i}-\frac{\left.c_{i}\left(\mathbb{E}\left[\theta_{i} \mid \Omega_{i}\right]+\eta_{i}+b_{i}-\theta_{i}-\eta_{i}\right)^{2}\right]}{2}\right] \\
& =\mathbb{E}\left[P_{i}\right]-\frac{c_{i}}{2}\left[\left(\mathbb{E}\left[\theta_{i} \mid \Omega_{i}\right]+b_{i}-\theta_{i}\right)^{2}\right] \\
& =0-\frac{c_{i}}{2} \mathbb{E}\left[\left(\mathbb{E}\left[\theta_{i} \mid \Omega_{i}\right]-\theta_{i}\right)^{2}+\left(b_{i}\right)^{2}+2 b_{i}\left(\mathbb{E}\left[\theta_{i} \mid \Omega_{i}\right]-\theta_{i}\right)\right]
\end{aligned}
$$

Simplifying further, we have

$$
\begin{aligned}
U_{i} & =-\frac{c_{i}}{2}\left[\mathbb{E}\left[\left(\mathbb{E}\left[\theta_{i} \mid \Omega_{i}\right]-\theta_{i}\right)^{2}\right]+\left(b_{i}\right)^{2}+2 b_{i} \mathbb{E}\left(\mathbb{E}\left[\theta_{i} \mid \Omega_{i}\right]-\theta_{i}\right)\right] \\
& =-\frac{c_{i}}{2}\left[\mathbb{E}\left[\mathbb{E}\left[\left(\mathbb{E}\left[\theta_{i} \mid \Omega_{i}\right]-\theta_{i}\right)^{2} \mid \Omega_{i}\right]\right]+\left(b_{i}\right)^{2}+0\right] \\
& =-\frac{c_{i}}{2}\left[\mathbb{E}\left[\mathbb{V a r}\left[\theta_{i} \mid \Omega_{i}\right]\right]+\left(b_{i}\right)^{2}\right] \\
& =-\frac{c_{i}}{2}\left[\mathbb{V} \operatorname{Var}\left[\theta_{i} \mid \Omega_{i}\right]+\left(b_{i}\right)^{2}\right] .
\end{aligned}
$$


The derivation of utilities in the benchmark case of simultaneous reporting is analogous.

\section{A.8 Proof of Lemma 4}

Let us compare the utilities of the firm reporting the second in the sequential case, $\mathbb{E}\left[U_{2}\right]$, and in the benchmark case of simultaneous reporting, $\mathbb{E}\left[U_{2}^{B}\right]$. Recall that

$$
\begin{aligned}
\mathbb{E}\left[U_{2}\right] & =-\frac{c_{2}}{2}\left(b_{2}^{2}+\operatorname{Var}\left[\theta_{2} \mid s_{2}, r_{1}\right]\right), \\
\mathbb{E}\left[U_{2}^{B}\right] & =-\frac{c_{2}}{2}\left(\left(b_{2}^{B}\right)^{2}+\operatorname{Var}\left[\theta_{2} \mid s_{2}\right]\right) .
\end{aligned}
$$

In order for $\mathbb{E}\left[U_{2}^{B}\right]$ to be greater than $\mathbb{E}\left[U_{2}\right]$, we must have

$$
b_{2}^{2}-\left(b_{2}^{B}\right)^{2}>\operatorname{Var}\left[\theta_{2} \mid s_{2}\right]-\operatorname{Var}\left[\theta_{2} \mid s_{2}, r_{1}\right] .
$$

Here,

$$
\begin{aligned}
\operatorname{Var}\left[\theta_{2} \mid s_{2}, r_{1}\right] & <\mathbb{V a r}\left[\theta_{2} \mid s_{2}\right], \\
b_{2} & >b_{2}^{B} \text { if and only if } \tau_{2}^{\eta}>\bar{\tau}_{2}^{\eta},
\end{aligned}
$$

and, consequently, the first necessary condition for $\mathbb{E}\left[U_{2}^{B}\right]$ to be greater than $\mathbb{E}\left[U_{2}\right]$ is to have $\tau_{2}^{\eta}>\bar{\tau}_{2}^{\eta}$, where $\bar{\tau}_{2}^{\eta}$ is defined in Theorem 1. Further, substitute the biases from (10) to have

$$
\begin{aligned}
& b_{2}^{2}-\left(b_{2}^{B}\right)^{2}>\operatorname{Var}\left[\theta_{2} \mid s_{2}\right]-\operatorname{Var}\left[\theta_{2} \mid s_{2}, r_{1}\right] \\
\Longleftrightarrow & \left(\frac{L_{22}\left(D_{1}, D_{2}\right)}{c_{2} D_{2}}\right)^{2}-\left(\frac{L_{22}\left(D_{1}^{B}, D_{2}^{B}\right)}{c_{2} D_{2}^{B}}\right)^{2}>\operatorname{Var}\left[\theta_{2} \mid s_{2}\right]-\operatorname{Var}\left[\theta_{2} \mid s_{2}, r_{1}\right] \\
\Longleftrightarrow & c_{2}<\frac{\left(\frac{L_{22}\left(D_{1}, D_{2}\right)}{D_{2}}\right)^{2}-\left(\frac{L_{22}\left(D_{1}^{B}, D_{2}^{B}\right)}{D_{2}^{B}}\right)^{2}}{\operatorname{Var}\left[\theta_{2} \mid s_{2}\right]-\operatorname{Var}\left[\theta_{2} \mid s_{2}, r_{1}\right]}
\end{aligned}
$$

Both the numerator and the denominator are positive and independent of $c_{2}$, so this ratio represents the $\bar{c}_{2}$ that makes the second condition.

\section{A.9 Proof of Theorem 2}

This is a direct corollary of Lemma 4.

\section{A.10 Proof of Proposition 3}

Recall the formulae for the biases in (10). In the case of simultaneous reporting, the weights that managers put on their own signals, $D_{i}^{B}, i=1,2$ do not depend on $\rho$. Consequently, we 
do not have to substitute $D_{1}^{B}$ and $D_{2}^{B}$ when taking the derivatives. It is straightforward to compute $\frac{d b_{i}^{B}}{d \rho}, i=1,2$ and see that the derivatives are negative.

In contrast, when computing the derivatives in the sequential setup we need to recall that $D_{2}$ depends on $\rho$, so that

$$
\frac{d b_{i}}{d \rho}=\underbrace{\frac{\partial b_{i}}{\partial \rho}}_{<0}+\underbrace{\frac{\partial b_{i}}{\partial D_{2}} \frac{\partial D_{2}}{\partial \rho}}_{<0}, i=1,2 .
$$

Substituting $D_{2}$ and computing the derivative of the follow manager bias, $\frac{d b_{2}}{d \rho}$, we find that the derivative remains negative in the sequential regime, In contrast, the derivative of the lead manager's bias is shown to have the same sign as

$$
M \tau_{2}^{\eta}+N
$$

where $M$ and $N$ are independent of $\tau_{2}^{\eta}$ and

$$
\begin{aligned}
M & =-\tau_{2}^{\varepsilon}\left(\tau_{2}^{\theta}+\tau_{2}^{\varepsilon}\right)\left(\left(\tau_{1}^{\theta}\right)^{3}+\left(\tau_{1}^{\varepsilon}\right)^{2}\left(\left(1-\rho^{2}\right) \tau_{1}^{\eta}+\tau_{1}^{\theta}\right)+\tau_{1}^{\theta} \tau_{1}^{\varepsilon}\left(2 \tau_{1}^{\theta}+\tau_{1}^{\eta}\right)\right){ }^{3}<0, \\
N & =L\left(K \rho^{2}-O\right)
\end{aligned}
$$

Here, $K, L$ and $O$ are positive, and $K$ and $O$ do not depend on $\rho$. We omit the formulae here in the interest of space. One can see that $M \tau_{2}^{\eta}+N$ is positive as long as $N$ is positive and $\tau_{2}^{\eta}<N / M$. Moreover, $N$ is positive as long as $K \rho^{2}>O$. This is attained when $K>O$ and $\rho>\sqrt{\frac{O}{K}}$ (Indeed, if $K \leq O$, then there is no such $\rho \in[-1,1]$ that $\left.K \rho^{2}>O\right)$.

We are left with establishing when $K>O$. This is true as long as

$$
-I \tau_{2}^{\varepsilon}+J>0
$$

where $I$ and $J$ are independent of $\tau_{2}^{\varepsilon}$ and we have

$$
\begin{aligned}
& I=\left(\tau_{1}^{\theta}\right)^{2}\left(\left(\tau_{1}^{\theta}\right)^{2}+\tau_{1}^{\varepsilon}\left(\tau_{1}^{\eta}+\tau_{1}^{\varepsilon}\right)+2 \tau_{1}^{\theta} \tau_{1}^{\varepsilon}\right)^{2}>0 \\
& J=\tau_{2}^{\theta}\left(\tau_{1}^{\varepsilon}+\tau_{1}^{\theta}\right)\left(\tau_{1}^{\theta}\left(\tau_{1}^{\varepsilon}+\tau_{1}^{\theta}\right)+\tau_{1}^{\varepsilon} \tau_{1}^{\eta}\right)\left(\tau_{1}^{\eta} \tau_{1}^{\varepsilon}\left(2 \tau_{1}^{\varepsilon}-\tau_{1}^{\theta}\right)-\left(\tau_{1}^{\varepsilon}\right)^{2} \tau_{1}^{\theta}-2 \tau_{1}^{\varepsilon}\left(\tau_{1}^{\theta}\right)^{2}-\left(\tau_{1}^{\theta}\right)^{3}\right) .
\end{aligned}
$$

For $-I \tau_{2}^{\varepsilon}+J>0$ to hold we need that $J>0$ and $\tau_{2}^{\varepsilon}<\frac{J}{I}$. For $J>0$ we need to have that

$$
\left(\tau_{1}^{\eta} \tau_{1}^{\varepsilon}\left(2 \tau_{1}^{\varepsilon}-\tau_{1}^{\theta}\right)-\left(\tau_{1}^{\varepsilon}\right)^{2} \tau_{1}^{\theta}-2 \tau_{1}^{\varepsilon}\left(\tau_{1}^{\theta}\right)^{2}-\left(\tau_{1}^{\theta}\right)^{3}\right)>0
$$

which holds when $\tau_{1}^{\varepsilon}>\tau_{1}^{\theta} / 2$ and that $\tau_{1}^{\eta}>\frac{\left(\tau_{1}^{\varepsilon}\right)^{2} \tau_{1}^{\theta}+2 \tau_{1}^{\varepsilon}\left(\tau_{1}^{\theta}\right)^{2}+\left(\tau_{1}^{\theta}\right)^{3}}{\tau_{1}^{\varepsilon}\left(2 \tau_{1}^{\varepsilon}-\tau_{1}^{\theta}\right)}$.

Summing up, we have the following necessary and sufficient conditions for $\frac{d b_{1}}{d \rho}$ to be 
positive:

$$
\begin{aligned}
\tau_{2}^{\eta}<T_{2}^{\eta}, \text { where } T_{2}^{\eta} & =N / M, \\
\rho<T^{\rho}, \text { where } T^{\rho} & =\sqrt{\frac{O}{K}} \\
\tau_{2}^{\varepsilon}<T_{2}^{\varepsilon}, \text { where } T_{2}^{\varepsilon} & =\frac{J}{I} \\
\tau_{1}^{\varepsilon}>T_{1}^{\varepsilon}, \text { where } T_{1}^{\varepsilon} & =\tau_{1}^{\theta} / 2, \\
\tau_{1}^{\eta}>T_{1}^{\eta}, \text { where } T_{1}^{\eta} & =\frac{\left(\tau_{1}^{\varepsilon}\right)^{2} \tau_{1}^{\theta}+2 \tau_{1}^{\varepsilon}\left(\tau_{1}^{\theta}\right)^{2}+\left(\tau_{1}^{\theta}\right)^{3}}{\tau_{1}^{\varepsilon}\left(2 \tau_{1}^{\varepsilon}-\tau_{1}^{\theta}\right)} .
\end{aligned}
$$

\section{A.11 Proof of Proposition 4}

When evaluating $b_{1}-b_{2}$ and $b^{B}-b_{2}$, we can see that the first difference, $b_{1}-b_{2}$, has the same sign as

$$
A\left(\tau^{\eta}\right)^{2}+B \tau^{\eta}+C,
$$

while the second difference, $b^{B}-b_{2}$, has the same sign as

$$
D\left(\tau^{\eta}\right)^{2}+E
$$

where

$$
\begin{aligned}
& A=-\left(\tau^{\varepsilon}\right)^{2}\left(\tau^{\varepsilon}\left(1-\rho^{2}\right)+\tau^{\theta}\right)^{2}<0, \\
& B=\rho^{2}\left(\tau^{\varepsilon}\right)^{2}\left(\tau^{\theta}\right)^{2}\left(\tau^{\varepsilon}+\tau^{\theta}\right)>0 \\
& C=\left(\tau^{\theta}\right)^{2}\left(\tau^{\varepsilon}+\tau^{\theta}\right)^{4}>0 \\
& D=-\left(\tau^{\varepsilon}\right)^{2}\left(\tau^{\varepsilon}\left(1-\rho^{2}\right)+\tau^{\theta}\right)<0 \\
& E=\left(\tau^{\theta}\right)^{2}\left(\tau^{\varepsilon}+\tau^{\theta}\right)^{3}>0 .
\end{aligned}
$$

Because $A<0$ and $C>0$, we have that $A\left(\tau^{\eta}\right)^{2}+B \tau^{\eta}+C>0$ when $\tau^{\eta}<\tau_{I I}^{\eta}$, where $\tau_{I I}^{\eta}$ is the largest root of equation $A x^{2}+B x+C=0$. Similarly, because $D<0$ and $E>0$, we have that $D\left(\tau^{\eta}\right)^{2}+E>0$ when $\tau^{\eta}<\tau_{I}^{\eta}$, where $\tau_{I}^{\eta}$ is the largest root of equation $D x^{2}+E=0$, i.e. $\tau_{I}^{\eta}=\sqrt{E /(-D)}$. From Theorem 1 , we know that $b_{1}>b^{B}$ always.

To conclude the proof, we need to show that $\tau_{I}^{\eta}<\tau_{I I}^{\eta}$. For this to hold it is enough to show that $A\left(\tau_{I}^{\eta}\right)^{2}+B \tau_{I}^{\eta}+C>0$. Substituting $\left(\tau_{I}^{\eta}\right)^{2}=E /(-D)$, we have

$$
A\left(\tau_{I}^{\eta}\right)^{2}+B \tau_{I}^{\eta}+C=-A E / D+B \tau_{I}^{\eta}+C=(A E-C D) /(-D)+B \tau_{I}^{\eta} .
$$

Simplifying $A E-C D$ and observing that it is always positive, we conclude the proof. 


\section{A.12 Proof of Propositions 5 and 6}

The proof is straightforward as the biases $b_{1}$ and $b_{2}$ as well as the reporting coefficients $D_{1}$, $D_{2}$ and $X$ are expressed in closed form.

\section{A.13 Proof of Proposition 7}

The coefficients are expressed in closed form in Lemma lemma:PRC. Taking derivatives, we see that the only derivative that can be nonnegative is $\frac{d A_{11}}{d \rho}$. This derivative has the same sign as

$$
A \rho^{4}+B \rho^{2}+C
$$

where

$$
\begin{aligned}
& A=-\left(\tau_{1}^{\varepsilon}\right)^{4}\left(\tau_{1}^{\eta}\right)^{2} \tau_{2}^{\varepsilon}\left(\tau_{2}^{\eta}+\tau_{2}^{\theta}\right) \\
& B=2\left(\tau_{1}^{\varepsilon}\right)^{2} \tau_{2}^{\eta}\left(\tau_{1}^{\varepsilon}+\tau_{1}^{\theta}\right)\left(\left(\tau_{1}^{\theta}\right)^{2}+\tau_{1}^{\varepsilon}\left(\tau_{1}^{\eta}+\tau_{1}^{\theta}\right)\right)\left(\left(\tau_{2}^{\theta}\right)^{2}+\tau_{2}^{\varepsilon}\left(\tau_{2}^{\eta}+\tau_{2}^{\theta}\right)\right) \\
& C=-\left(\tau_{1}^{\varepsilon}+\tau_{1}^{\theta}\right)^{2}\left(\left(\tau_{1}^{\theta}\right)^{2}+\tau_{1}^{\varepsilon}\left(\tau_{1}^{\eta}+\tau_{1}^{\theta}\right)\right)^{2}\left(\left(\tau_{2}^{\theta}\right)^{2}+\tau_{2}^{\varepsilon}\left(\tau_{2}^{\eta}+\tau_{2}^{\theta}\right)\right) .
\end{aligned}
$$

One can see that this is a quadratic function of $\rho^{2}$. We can establish that $-B /(2 A)>1$, and consequently, there are two possible cases. Either $A+B+C<0$ and then $\frac{d A_{11}}{d \rho}<0$, or $A+B+C>0$, then there exists $K^{\rho} \in[0,1]$ such that for $\rho<K^{\rho}$ we have $\frac{d A_{11}}{d \rho}<0$ and for $\rho>K^{\rho}$ we have $\frac{d A_{11}}{d \rho}<0$, where $\left(K^{\rho}\right)^{2}$ is the lowest root of the equation $A x^{2}+B x+C=0$.

To find the conditions for $A+B+C>0$, observe that

$$
A+B+C=N-M \tau_{2}^{\varepsilon}
$$

where

$$
\begin{aligned}
& M=\left(\tau_{2}^{\theta}\right)^{2}\left(\tau_{1}^{\varepsilon}+\tau_{1}^{\theta}\right)\left(\left(\tau_{1}^{\theta}\right)^{2}+\tau_{1}^{\varepsilon}\left(\tau_{1}^{\eta}+\tau_{1}^{\theta}\right)\right)>0 \\
& N=\left(\tau_{1}^{\theta}\right)^{2}\left(\tau_{2}^{\eta}+\tau_{2}^{\theta}\right)\left(\tau_{1}^{\varepsilon}\left(\tau_{1}^{\eta}+\tau_{1}^{\varepsilon}\right)+\tau_{1}^{\theta}\left(2 \tau_{1}^{\varepsilon}+\tau_{1}^{\theta}\right)\right)^{2}\left(\tau_{1}^{\eta} \tau_{1}^{\varepsilon}\left(\tau_{1}^{\varepsilon}-\tau_{1}^{\theta}\right)-\tau_{1}^{\theta}\left(\tau_{1}^{\varepsilon}+\tau_{1}^{\theta}\right)^{2}\right)
\end{aligned}
$$

One can see that in order for $A+B+C$ to be greater than zero, one need to have $N>0$ and $\tau_{2}^{\varepsilon}<N / M$. In order to see the conditions for $N>0$, we consider the last factor of $N$ in the equation above. This factor is positive as long as $\tau_{1}^{\varepsilon}>\tau_{1}^{\theta}$ and $\tau_{1}^{\eta}>\frac{\tau_{1}^{\theta}\left(\tau_{1}^{\varepsilon}+\tau_{1}^{\theta}\right)^{2}}{\tau_{1}^{\varepsilon}\left(\tau_{1}^{\varepsilon}-\tau_{1}^{\theta}\right)}$.

Summing up, we have the following necessary and sufficient conditions for $\frac{d A_{11}}{d \rho}$ to be 
positive:

$$
\begin{aligned}
\rho & <K^{\rho}, \text { where }\left(K^{\rho}\right)^{2} \text { is the lowest root of the equation } A x^{2}+B x+C=0, \\
\tau_{2}^{\varepsilon} & <K_{2}^{\varepsilon}, \text { where } K_{2}^{\varepsilon}=\frac{N}{M}, \\
\tau_{1}^{\varepsilon} & >K_{1}^{\varepsilon} \text {, where } K_{1}^{\varepsilon}=\tau_{1}^{\theta}, \\
\tau_{1}^{\eta} & >K_{1}^{\eta}, \text { where } K_{1}^{\eta}=\frac{\tau_{1}^{\theta}\left(\tau_{1}^{\varepsilon}+\tau_{1}^{\theta}\right)^{2}}{\tau_{1}^{\varepsilon}\left(\tau_{1}^{\varepsilon}-\tau_{1}^{\theta}\right)} .
\end{aligned}
$$

\section{A.14 Proof of Proposition 8}

Let us start with proving that

$$
A_{22}>A_{22}^{B} \Leftrightarrow A_{12}>A_{12}^{B} \Leftrightarrow b_{2}>b_{2}^{B} \Leftrightarrow \tau_{2}^{\eta}>\bar{\tau}_{2}^{\eta} .
$$

We showed in Theorem 1 that

$$
b_{2}>b_{2}^{B} \Leftrightarrow \tau_{2}^{\eta}>\bar{\tau}_{2}^{\eta} .
$$

Since $b_{2}=\frac{A_{22}}{c_{2}}$ and $b_{2}^{B}=\frac{A_{22}^{B}}{c_{2}}$, we have that

$$
A_{22}>A_{22}^{B} \Leftrightarrow b_{2}>b_{2}^{B} .
$$

It is left to show

$$
A_{22}>A_{22}^{B} \Leftrightarrow A_{12}>A_{12}^{B} .
$$

Observe that

$$
\begin{aligned}
A_{12}-A_{12}^{B} & =\frac{L_{12}}{D_{2}}-\frac{L_{12}^{B}}{D_{2}^{B}} \\
& =\frac{L_{12} D_{2}^{B}-L_{12}^{B} D_{2}}{D_{2} D_{2}^{B}}
\end{aligned}
$$

Further, we derive that

$$
\frac{L_{12}}{L_{22}}=\frac{L_{12}^{B}}{L_{22}^{B}}=\alpha
$$

where $\alpha=-\frac{\left.\hat{\Sigma}_{11}\left(\Sigma_{\theta}^{-1}\right)\right|_{12}}{\left.\hat{\Sigma}_{11}\left(\Sigma_{\theta}^{-1}\right)\right|_{11}+1}$ anf $\alpha>0$ because $\hat{\Sigma}_{11}>0,\left.\left(\Sigma_{\theta}^{-1}\right)\right|_{11}>0$ and $\left.\left(\Sigma_{\theta}^{-1}\right)\right|_{12}<0$. It follows that,

$$
\begin{aligned}
A_{12}-A_{12}^{B} & =\frac{L_{12} D_{2}^{B}-L_{12}^{B} D_{2}}{D_{2} D_{2}^{B}} \\
& =\alpha \frac{L_{22} D_{2}^{B}-L_{22}^{B} D_{2}}{D_{2} D_{2}^{B}} \\
& =\alpha\left(A_{22}-A_{22}^{B}\right)
\end{aligned}
$$


and, consequently,

$$
A_{22}>A_{22}^{B} \Leftrightarrow A_{12}>A_{12}^{B} .
$$

Similarly, we find the conditions for which

$$
A_{11}-A_{11}^{B} \Leftrightarrow A_{21}>A_{21}^{B}
$$

We show that $A_{11}>A_{11}^{B}$ has the same sign as

$$
-M+\tau_{1}^{\eta}\left(-N+\rho^{2} O\right),
$$

where

$$
\begin{aligned}
M & =\tau_{1}^{\theta}\left(\tau_{1}^{\varepsilon}+\tau_{1}^{\theta}\right)^{2}\left(\left(\tau_{2}^{\theta}\right)^{2}+\tau_{2}^{\varepsilon}\left(\tau_{2}^{\eta}+\tau_{2}^{\theta}\right)\right)>0, \\
L & =\tau_{1}^{\theta}\left(\tau_{1}^{\varepsilon}+\tau_{1}^{\theta}\right)\left(\left(\tau_{2}^{\theta}\right)^{2}+\tau_{2}^{\varepsilon}\left(\tau_{2}^{\eta}+\tau_{2}^{\theta}\right)\right)>0, \\
O & =\left(\tau_{2}^{\varepsilon}\right)^{2}\left(\left(\tau_{2}^{\theta}\right)^{2}+\tau_{2}^{\varepsilon}\left(\tau_{2}^{\eta}+2 \tau_{2}^{\theta}\right)\right)>0 .
\end{aligned}
$$

Further, we have that

$$
-L+O=\tau_{1}^{\varepsilon}\left(\tau_{1}^{\varepsilon} \tau_{2}^{\varepsilon} \tau_{2}^{\theta}-\tau_{1}^{\theta}\left(\left(\tau_{2}^{\theta}\right)^{2}+\tau_{2}^{\varepsilon}\left(\tau_{2}^{\eta}+\tau_{2}^{\theta}\right)\right)\right) .
$$

In order to have $-M+\tau_{1}^{\eta}\left(-L+\rho^{2} O\right)>0$ we need first to ensure that $-L+O>0$, i.e.

$$
\tau_{1}^{\theta}<N_{1}^{\theta}=\frac{\tau_{1}^{\varepsilon} \tau_{2}^{\varepsilon} \tau_{2}^{\theta}}{\left(\tau_{2}^{\theta}\right)^{2}+\tau_{2}^{\varepsilon}\left(\tau_{2}^{\eta}+\tau_{2}^{\theta}\right)} .
$$

If this hold then there exist $\rho>N^{\rho}=\sqrt{\frac{L}{O}}$ for which $\left(-L+\rho^{2} O\right)>0$. Finally, if

$$
\tau_{1}^{\eta}>N_{1}^{\eta}=\frac{M}{-L+\rho^{2} O},
$$

then $-M+\tau_{1}^{\eta}\left(-L+\rho^{2} O\right)>0$ and $A_{11}>A_{11}^{B}$. If one of these three conditions is not satisfied then $-M+\tau_{1}^{\eta}\left(-L+\rho^{2} O\right)<0$ and $A_{11}<A_{11}^{B}$.

\section{A.15 Proof of Proposition 9}

Recall that the first report in the sequential scenario is given by

$$
r_{1}=D_{1} s_{1}+\eta_{1}+\frac{A_{11}}{c_{1}} .
$$


Conditional upon observing $r_{1}$, the risk neutral investors price the firms linearly by computing Bayesian updates, so that

$$
P_{1}^{0}=\mathbb{E}\left[\theta_{1} \mid r_{1}\right]=\frac{\frac{r_{1}-A_{11} / c_{1}}{D_{1}} \frac{1}{1 / \tau_{1}^{\varepsilon}+1 /\left(D_{1}^{2} \tau_{1}^{\eta}\right)}+\mathbb{E}\left[\theta_{1}\right] \tau_{1}^{\theta}}{\frac{1}{1 / \tau_{1}^{\varepsilon}+1 /\left(D_{1}^{2} \tau_{1}^{\eta}\right)}+\tau_{1}^{\theta}},
$$

where $\mathbb{E}\left[\theta_{1}\right]=0$. Extracting the coefficient in front of $r_{1}$, we obtain

$$
A_{1}^{0}=\frac{1}{D_{1}} \frac{1}{1+\tau_{1}^{\theta} / \tau_{1}^{\varepsilon}+\tau_{1}^{\theta} /\left(D_{1}^{2} \tau_{1}^{\eta}\right)} .
$$

The rest of the proof is a straightforward comparison of the coefficients, all of which are expressed in closed form.

\section{A.16 Proof of Proposition 10}

Recall from Proposition 9 that

$$
A_{1}^{0}=\frac{1}{D_{1}} \frac{1}{1+\tau_{1}^{\theta} / \tau_{1}^{\varepsilon}+\tau_{1}^{\theta} /\left(D_{1}^{2} \tau_{1}^{\eta}\right)} .
$$

We prove by substituting $L_{11}\left(D_{1}, D_{2}\right)$ : it turns out that

$$
A_{1}^{0}-\frac{L_{11}}{D_{1}}>0
$$

for any $D_{1}>0$ and $D_{2}>0$.

\section{B Intuition: Single firm case}

In this appendix, we consider a single-firm setting to develop additional intuition for multifirm results in Section 3. First, consider a report $r=X s+\frac{X}{I} \eta$, where $s=\theta+\varepsilon$ and $\eta$ are distributed normally. The mean of each of these random variables is zero and the precisions of $\theta, \varepsilon$ and $\eta$ are $\tau^{\theta}, \tau^{\varepsilon}$ and $\tau^{\eta}$, respectively. Let us call $X>0$ the scaling factor of the report $r$ and $I>0$ the informativeness factor of the report $r$. Observe that $X$ captures the scaling of the report but does not affect its information content. The posterior variance of $\theta$ is given by

$$
\operatorname{Var}(\theta \mid r)=\frac{1}{\tau^{\theta}+\frac{1}{\frac{1}{\tau^{\varepsilon}}+\frac{1}{\tau^{\eta} I^{2}}}} .
$$

This variance (and thus the informativeness of the report) is not affected by the scaling factor $X$, but increases in the informativeness factor $I$. 
We can derive that

$$
\begin{aligned}
E[\theta \mid r] & =E[\theta]+\frac{\operatorname{Cov}(r, \theta)}{\operatorname{Var}(r)}(r-E[r])=\frac{\operatorname{Cov}(r, \theta)}{\operatorname{Var}(r)} r \\
& =\frac{X \operatorname{Var}(\theta)}{X^{2} \operatorname{Var}(\theta)+X^{2} \operatorname{Var}(\varepsilon)+\frac{X^{2}}{I^{2}} \operatorname{Var}(\eta)} r=\frac{\operatorname{Var}(\theta)}{X \operatorname{Var}(\theta)+X \operatorname{Var}(\varepsilon)+\frac{X}{I^{2}} \operatorname{Var}(\eta)} r .
\end{aligned}
$$

One can see that a higher informativeness factor $I$ increases the weight put on the report (because the information content increases), while a higher scaling factor $X$ decreases the weight put on the report (because the report is larger, while the information content is not affected by $X)$ :

$$
\frac{d E[\theta \mid r]}{d I}>0, \frac{d E[\theta \mid r]}{d X}<0
$$

Let us consider the normalized report $\tilde{r}=\frac{r}{X}$. Observe that the weight put on the normalized report essentially has only the informativeness factor in it, while the scaling goes away:

$$
\begin{aligned}
E[\theta \mid r] & =\frac{\operatorname{Var}(\theta)}{X \operatorname{Var}(\theta)+X \operatorname{Var}(\varepsilon)+\frac{X}{I^{2}} \operatorname{Var}(\eta)} r \\
& =\frac{\operatorname{Var}(\theta)}{\operatorname{Var}(\theta)+\operatorname{Var}(\varepsilon)+\frac{1}{I^{2}} \operatorname{Var}(\eta)} \tilde{r}=E[\theta \mid \tilde{r}] .
\end{aligned}
$$

In this type of problem normalizing the report by $X$ essentially undoes the scaling factor and isolates the informativeness factor.

Now let us go back to our problem. We have a signal

$$
r=D s+\eta
$$

which can be rewritten in the form $r=X s+\frac{X}{I} \eta$ for $X=D$ and $I=D$. One can see that the same factor $D$ captures both the scaling and the informativeness, so that the derivative is not monotonic. Indeed,

$$
\frac{d E[\theta \mid r]}{d D}=\left.\left(\frac{\partial E[\theta \mid r]}{\partial X}+\frac{\partial E[\theta \mid r]}{\partial I}\right)\right|_{X=D, I=D}
$$

where the first derivative is negative and the second one is positive.

How should we disentangle the informativeness effect from the scaling effect? We normalize the signal and consider the signal $\tilde{r}=\frac{r}{X}=s+\frac{1}{I} \eta=s+\frac{1}{D} \eta$. This procedure allows to get rid of the scaling effect and isolate the informativeness effect. This corresponds to the market updating discussed in Section 3. 